IBL- -31144

DE93 001601

\title{
THE USE OF ENERGY MANAGEMENT AND CONTROL SYSTEMS FOR RETROFIT PERFORMANCE MONITORING IN THE LOANSTAR PROGRAM
}

\author{
Final Report \\ April 1992 \\ prepared for: \\ Texas Governor's Energy Management Center \\ Texas A\&M University \\ prepared by: \\ Kristin E. Heinemeier and Hashem Akbari \\ Energy Analysis Program \\ Energy and Environment Division \\ Lawrence Berkeley Laboratory \\ Berkeley, California 94720 \\ David Claridge, Jeff Haberl, Brian Poynor, and Raghu Belur \\ Energy Systems Laboratory \\ Department of Mechanical Engineering \\ Texas Engineering Experiment Station \\ Texas A\&M University \\ College Station, Texas 77843
}

This work was sponsored by the Texas Governor's Energy Management Center, as part of the LoanSTAR Monitoring and Analysis Project, through Texas A\&M University (Award No. BG90319); and by the Assistant Secretary for Conservation and Renewable Energy, Office of Building Technology of the U.S. Department of Energy, under Contract No. DE-AC03-76SF00098. 


\begin{abstract}
Monitoring of building energy consumption, operation, and weather variables are important parts of retrofit evaluation projects. However, effective monitoring can be quite expensive. In this report, we investigate the feasibility of using a building's existing energy management and control system (EMCS) for gathering some or all of this required data, rather than installing dedicated data-logging equipment. Three case studies in the Texas LoanSTAR retrofit monitoring program were used to explore the current applicability of existing EMCSs for monitoring, and to identify areas requiring additional development. It was found that data could be retrieved in each case; without installing any additional hardware or software in two of the three sites, and with the addition of only a few sensors and a minor software modification in the third. However, the process of using the EMCS for monitoring was inconvenient in several respects. It was determined that the process could be greatly simplified if EMCS manufacturers ensured that their software averaged data over an hourly interval and reliably reported them at the end of each hour, used concise and consistent formats for requesting and reporting the data, and provided a simple means of displaying or transmitting the data.
\end{abstract}




\section{INTRODUCTION AND MOTIVATION}

The Texas LoanSTAR program is a revolving loan program supported by the state of Texas's oil overcharge funds. Loans are used to fund energy conservation retrofits in state and local government facilities, and are paid back from energy savings. The program also has a strong emphasis on evaluating the performance of the retrofits. Initial estimates of projected energy savings are often inaccurate, and the LoanSTAR program emphasizes monitoring of actual energy consumption in order to determine savings. The monitoring includes acquiring facility or whole-building monthly utility data, hourly whole-building data, and hourly submetered data.

In the LoanSTAR program, as well as in most other monitoring programs, most of the hourly data acquisition is done by installing dedicated monitoring equipment, i.e., equipment whose sole purpose is to perform monitoring for the project. Dedicated monitoring equipment must include several different types of devices (summarized in Table 1). The first, of course, are the sensors needed to measure the required quantities. Once the sensors have been installed, wiring must be installed to connect the sensors to the datalogger. The datalogger includes software to sample, condition, average, and store the data. There must also be storage devices: short term, volatile memory, and possibly a longer term permanent storage device, such as a magnetic-tape drive. The data can often be retrieved remotely, requiring a modem and an algorithm for downloading the data. All of this equipment, hardware and software, must be purchased.

Upon reviewing this list of equipment required for monitoring, one will recognize that most Energy Management and Control Systems (EMCSs), used primarily to control building systems, include these same devices. An EMCS can include thousands of sensors; sophisticated interconnections between sensors, actuators, and processors (often comprising a local area network); a powerful and fast processor capable of extensive computation; megabytes of memory; a large variety of available peripheral storage devices; and a modem and communications capabilities. Since equipment required for monitoring is likely to be present at a site, it would seem advantageous to make use of it in a monitoring project. Table 1 illustrates some of these advantages. However, since the EMCS was designed for control of the building systems, its application in monitoring may have several disadvantages (also discussed in Table 1) and often several details have to be worked out to apply it most conveniently.

In this project, we used three case study facilities in the LoanSTAR program to investigate how existing EMCSs can be applied to remote monitoring, and to determine what limitations exist and what areas require further development in order to encourage more frequent use of the technology. This report summarizes that investigation. First, it discusses generally how EMCSs are used for monitoring, and how monitoring was usually performed in the LoanSTAR case studies. Then it discusses some of the unique advantages of EMCS monitoring over dedicated monitoring. It then discusses each case study site in detail, starting with a discussion of the building, retrofit, and general EMCS characteristics. The relevant EMCS-monitoring characteristics (data measurement, storage, access, and proressing) are then discussed for each site. Finally, the process of monitoring in these sites is evaluated, and general conclusions are made concerning the strengths and weaknesses of EMCS monitoring, and the changes that should be made to make the process more convenient. 
TABLE 1-Comparison of Dedicated and EMCS-Based Monitoring Components.

\begin{tabular}{|c|c|c|}
\hline & $\begin{array}{l}\text { Dedicated } \\
\text { Monitoring }\end{array}$ & EMCS \\
\hline Sensors & $\begin{array}{l}\text { Must be purchased } \\
\text { Must be installed } \\
\text { Researcher can select type } \\
\text { Researcher can select accuracy } \\
\text { Researcher can calibrate }\end{array}$ & $\begin{array}{l}\text { Existing } \\
\text { Numerous points } \\
\text { Used for building control } \\
\text { May have to add required sen- } \\
\text { sors } \\
\text { Accuracy and calibration not } \\
\text { under researcher's control }\end{array}$ \\
\hline Connections & $\begin{array}{l}\text { Must be purchased } \\
\text { Must be installed } \\
\text { No other traffic } \\
\text { Can be sized for monitoring }\end{array}$ & $\begin{array}{l}\text { Existing } \\
\text { Sophisticated networking } \\
\text { Other traffic can interfere with } \\
\text { monitoring } \\
\text { Monitoring traffic can interfere } \\
\text { with operation } \\
\text { Must be added if expansion } \\
\text { needed }\end{array}$ \\
\hline Data Logging Processor & $\begin{array}{l}\text { Must be purchased } \\
\text { Simple processor }\end{array}$ & $\begin{array}{l}\text { Existing } \\
\text { Sophisticated processor }\end{array}$ \\
\hline Data Logging Software & $\begin{array}{l}\text { Must be purchased } \\
\text { Simple sampling, conditioning, } \\
\text { averaging } \\
\text { Predefined } \\
\text { Appropriate for monitoring } \\
\text { needs }\end{array}$ & $\begin{array}{l}\text { Existing } \\
\text { Often simple sampling, condi- } \\
\text { tioning, averaging } \\
\text { Often predefined } \\
\text { Not defined for monitoring } \\
\text { needs } \\
\text { Can be sophisticated computa- } \\
\text { tion or calculation }\end{array}$ \\
\hline Data Storage & $\begin{array}{l}\text { Must be purchased } \\
\text { Usually limited size }\end{array}$ & $\begin{array}{l}\text { Existing } \\
\text { Sometimes must be supple- } \\
\text { mented } \\
\text { Both short and long term } \\
\text { Sometimes size limitations }\end{array}$ \\
\hline Modem & $\begin{array}{l}\text { Must be purchased } \\
\text { Dedicated to researcher }\end{array}$ & $\begin{array}{l}\text { Existing } \\
\text { Also used by operator, vendor }\end{array}$ \\
\hline
\end{tabular}

\section{EMCS MONITORING}

Dedicated monitoring consists of four steps: measurement, storage, access, and processing. These same steps are used in EMCS monitoring, although they are somewhat altered since the monitoring equipment belongs to the building personnel, and must primarily serve their needs. 


\section{Data Measurement}

EMCSs monitor a variety of control points within a building. To do so, an EMCS is equipped with a large number of sensors. In most cases, these sensors are very similar to those used in dedicated monitoring projects, and can include power transducers or integrating energy meters, as well as temperature, pressure, and humidity sensors. An important figure, used to indicate the size or complexity of an EMCS, is the number of installed points. The term "points" refers to inputs (sensors), outputs (actuators, for example damper motors and valves), and intermediate values (for example setpoints).

The primary objectives for installing an EMCS are to facilitate better control and simplify day-to-day operation of the building, as weil as to minimize energy consumption. Sensors are added to meet the building's objectives, and not a monitoring project's objectives. Hence, while certain variables such as whole-building energy consumption may usually be measured, end-use submetered energy consumption typically is not. Existing sensors may not be of sufficient accuracy or reliability for the monitoring project's needs. Also, the sensors cannot be easily calibrated or verified by the researchers. Depending on the needs of the monitoring project, it is possible that the project can entirely make use of existing sensors, and require no installation of sensors.

\section{Data Storage}

Data collected for immediate use in control applications, (for example in a calculation to determine if more cooling is required), can be stored for further analysis. Stored data can be used to determine, for example, how much temperatures in a certain zone have been fluctuating over the past day, or how energy consumption has changed over the past year.

Because of the usefulness of this type of data for building operation, most EMCSs have a facility for storing large amounts of data, often called "trending." One must remember that trended data are stored and presented in a way that was determined by the manufacturer to be useful for building operators in running their facilities, not for building researchers, and the format may be less appropriate for the latter.

Trended data can be stored in several locations in the EMCS. Many EMCSs now have a distributed architecture (see Figure 1), with networked command modules, each connected to local modules; and the ability for a host computer to be connected in the network. A small amount of data can sometimes be stored on the local module, a larger amount of data can be stored on the command modules, and longterm storage of data can be done with the host computer, and its peripheral data storage media (hard or floppy disk or magnetic tape). The storage medium, the number of points that can be trended, and the amount of data that can be stored for each point vary quite a bit among different EMCSs, although in most cases the hardware capacity is sufficient for remote monitoring needs. With a distributed architecture, information other than that being trended for energy monitoring is travelling along the network paths, and one must consider both the impact of energy monitoring traffic on other operations, and the impact of other operations on energy data transmission. 


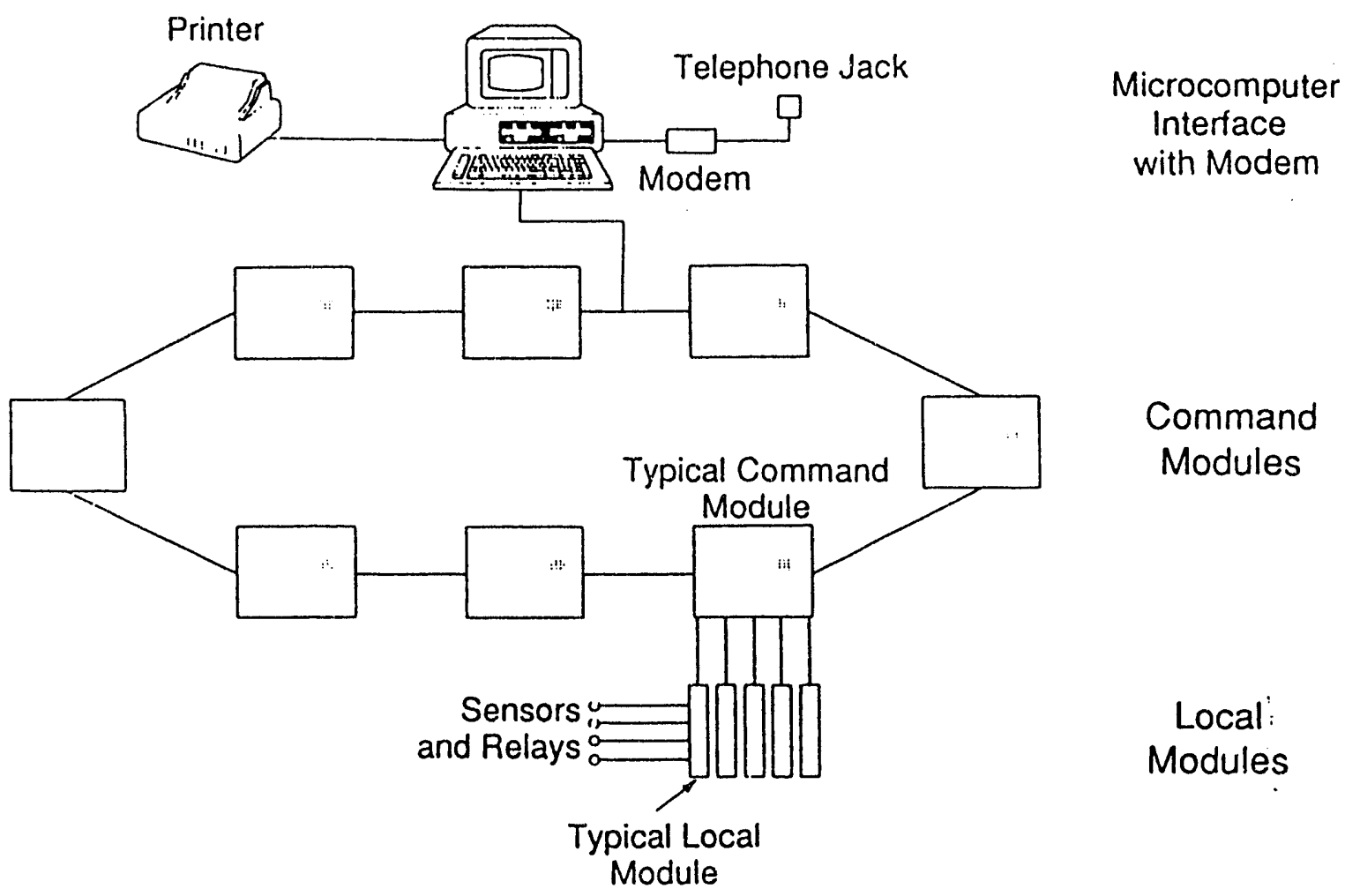

FIGURE 1-Architecture of a Typical Energy Management and Control System. Many EMCSs have a distributed architecture, allowing data to be stored or displayed at any of a number of locations, and allowing a remote computer to connect at any of a number of places. 
Data Access

EMCSs are often designed to present trended data in a graphical format or in a numerical report format. The EMCS operator can either look at the data report on the screen of the EMCS computer, print the data to a printer, or write the data to a computer file in order to later read it into another program, such as a spreadsheet or database program.

In order to access the data remotely, one can make use of the fact that most EMCSs allow for a remote computer to be tied into the system's network. This remote computer can either be a "dumb" terminal or a microcomputer, equipped with a modem and communications software, and communicating over commercial telephone lines. This remote computer is used to allow operators to check in on the system and control it from another location, or for vendors to trouble-shoot problems from their office. Most EMCSs include the required hardware for communications, and have a telephone line dedicated to the EMCS use, and these can usually be used by monitoring projects, so long as these monitoring activities do not tie up the equipment when it is needed for other tasks.

The procedure we used most often was to use an IBM PC compatible computer to dial up and connect to the EMCS's modem. Most commercial communications programs could be used to make this connection, and we used Procomm Plus, (or PCPLUS). Table 2 shows the procedure used to configure the communications program to connect to the EMCS, for PCPLUS as well as for Kermit and Crosstalk, two other commonly used communications programs. There are then two ways of transferring data: displaying a report on the remote computer's screen, and downloading the data file. In the first method, one uses the remote computer to log onto the EMCS system just as any other user, and run the trend utility, requesting that the data report be presented on the screen. The entire session is recorded in a log file on the remote computer, so that while the report is displayed on the screen, it is simultaneously recorded on the remote disk. In the second rnethod, the data are stored to an EMCS disk file, and transferred to the remote computer, using some kind of file transfer algorithm. The file transfer algorithm can either be embedded in the EMCS computer software, or can be implemented in a communications program, running in parallel with the EMCS software. If communications software is running in parallel, the EMCS must be on a computer with an operating system that allows multiple processes, and the asynchronous communications must not conflict with the more essential EMCS operations. Both procedures could be automated using a script file to watch for certain prompts coming from the EMCS, and to output the proper responses.

\section{Data Processing}

Data processing is necessary with both dedicated and EMCS monitoring, in order to translate data from different sources into a common format. In the case of EMCS monitoring, the relevant data are usually embedded in a lengthy text report, and the log files have to be processed to create data files. We wrote programs to process some of the data formats using a pattern scanning and processing language, AWK. This is a common UNIX utility, although PC versions are also available. 
TABLE 2-An Example of the Procedures for Setting Communications Parameters and Calling up an EMCS from a PC-Assuming Paremeters 1200-N-8-1.

\begin{tabular}{|c|c|c|}
\hline KERMIT & CROSSTALK & PCPLUS \\
\hline \multicolumn{2}{|l|}{ Start Program } & \multirow[b]{2}{*}{ peplus } \\
\hline kermit & xtalk & \\
\hline \multicolumn{3}{|c|}{ Setup Communications Parameters } \\
\hline \multirow[t]{2}{*}{ set baud 1200} & sp 1200 & ALT-p \\
\hline & & 2 \\
\hline \multirow[t]{3}{*}{ set parity none } & parity none & $\mathbf{n}$ \\
\hline & data 8 & ALT-8 \\
\hline & stop 1 & ALT-1 \\
\hline \multirow[t]{2}{*}{ set port 1} & port 1 & $\mathrm{~F} 1$ \\
\hline & & ESC \\
\hline \multicolumn{3}{|c|}{ Setup Terminal Emulation } \\
\hline \multirow[t]{4}{*}{ set terminal vt102 } & emulate DEC & ALT-s \\
\hline & & $\mathrm{t}$ \\
\hline & & $\begin{array}{l}\mathrm{g} \\
(\mathrm{vt102})\end{array}$ \\
\hline & & $\mathrm{ESC}$ \\
\hline \multicolumn{3}{|l|}{ Begin Logging Session } \\
\hline log session (filename) & ca (filename) & $\begin{array}{l}\text { ALT-F1 } \\
\text { (filename) }\end{array}$ \\
\hline \multicolumn{3}{|l|}{ Dial Phone } \\
\hline c & nu (phone number) & ALT-d \\
\hline AT & go & $\mathbf{m}$ \\
\hline ATDT (phone number) & & (phone number) \\
\hline \multicolumn{3}{|c|}{ EMCS Procedures-Unique to EMCS: Logging In, Requesting data, and Logging Out } \\
\hline \multicolumn{3}{|c|}{ Hangup and Exit Program } \\
\hline CTRL-] & HOME & ALT-x \\
\hline c & quit & y \\
\hline hangup & & y \\
\hline quit & & \\
\hline
\end{tabular}




\section{EMCS MONITORING ADVANTAGES}

The most obvious advantage of EMCS monitoring is that it can make use of existing hardware and software. With EMCS monitoring, it is often possible to make use entirely of existing equipment, and to install absolutely no hardware. Since no protocols exist for EMCS monitoring, each EMCS is a unique case, time will be required to learn how to use the existing capabilities, and coordination with building personnel. Time will also be required to ensure that the EMCS is capable of monitoring, and possibly for some reconfiguration. This time may be significant, although it may represent the only costs involved in starting up the monitoring. ${ }^{1}$ If EMCS monitoring becomes more routinely applied, and EMCS manufacturers begin designing their systems with remote monitoring in mind, the time and costs involved will be greatly reduced.

Another advantage of EMCS monitoring is that it is possible to obtain much more information about the building operation than is typically economically feasible in a dedicated monitoring project. In dedicated monitoring, the number of points that can be monitored is usually limited by the number of available input channels on the data logger, and the cost of hardwiring the connections from the sensors to the logger. With an EMCS, however, many points of interest are monitored in order to control the building, and the network connecting the sensors to the data storage medium is already in place.

The computational capabilities of EMCS processors are quite extensive and often underutilized. This provides the opportunity to perform basic processing of the data at the retrofit site, before the data are transmitted to the monitoring site. This kind of processing can include things like calculation of chilled water energy consumption or of heat recovery energy savings from flowrates and temperatures, and different kinds of averaging.

With access to several types of data, different types of analysis will also be possible. For example, the EMCS can match equipment hourly runtime with hourly energy consumption to obtain a more accurate representation of energy consumption during equipment operation. Or the status of motion detectors can be aggregated across the building, and used to automatically normalize energy consumption for occupancy.

\section{METHODOLOGY}

The method used to investigate the application of EMCSs to performance monitoring was to apply it in case studies. Several case studies have been performed in the past (see Akbari et al. 1989, Flcra et al. 1986, Heinemeier and Akbari 1987, and LeConiac et al. 1986), and the case study approach has been

\footnotetext{
1 It is difficult to estimate how much time was required to obtain data from the case study sites, due to the fact that our project was research oriented, and intended as a proof of concept rather than a realistic example of the implementation of this technology. However, as a rough estimate, it should not take more than the equivalent of two to three weeks of effort to make a system functional for monitoring (often much less-on the order of hours). At a rate of $\$ 40$ per hour, the three week effort would cost roughly $\$ 5000$, regardless of the number of points being monitored. If ten points are monitored, this corresponds to about $\$ 500$ per point. If 50 points are monitored, this corresponds to $\$ 100$ per point. As a comparison, dedicated monitoring can cost on the order of $\$ 1000$ per monitored point.
} 
found to be quite useful for this technology. Since remote energy monitoring has not been recognized as an application by EMCS manufacturers, each EMCS model, and in fact, each site investigated has provided unique challenges. By investigating several manufacturers, and several different sites, we begin to discover generic issues, and common limitations.

The three sites we investigated were all potentially LoanSTAR program participants (although one site was subsequently dropped from the program). All sites are college facilities in Texas (see Figure 2). Each site used a different brand of EMCS.

We report here on the steps that were required to initiate monitoring, the problems that were encountered, the solutions to those problems, and our evaluation of the entire process of obtaining data from the sites. We were able to obtain data from all three sites, and samples of those data are presented here as well.

\section{CASE STUDY 1: PRAIRIE VIEW A\&M}

\section{Building and EMCS}

The Prairie View A\&M (PVAM) campus, located near Houston, consists of about 46 buildings, and is shown in Figure 3. Several conservation measures have been recommended in many of the buildings. These conservation measures include lighting retrofits, variable speed chilled and hot water pumping, installation of a high efficiency base-load chiller, steam leak repairs, and controls to operate water well pumps during off-peak periods. These conservation measures are expected to result in energy cost savings of $\$ 298,000 /$ year (the campus' base year energy expenditures were about $\$ 2.1$ million), with an overall simple payback time of 2.9 years. The final list of retrofits has not yet been determined.

The PVAM campus is equipped with a Johnson Controls EMCS, model JC/85/40. The EMCS is connected to over 2000 points throughout the campus, including numerous $\mathrm{kWh}$ and Btu points. Table 3 illustrates the type of points that can be available for each air handling unit and each building.

Johnson Controls has a facility energy managenent contract with the campus. We were able to obtain information from Johnson Controls personnel at the site in Prairie View, at the regional office in Houston, and at the headquarters in Milwaukee. We visited the site and toured several buildings in June of 1990, and met with personnel at the site again in April 1991. We have received documentation on trending, as well as on the operation of the Extended Application Workstation (EAW), and the PC Distributed Data Base Manager (PCDDBM).

\section{Data Storage}

During our meetings, we were told that the system can trend up to 99 points, and can store up to 3000 samples for each point, using this system. However, this limit only applies to a JC/85/40 that is running on a PC. The JC/85/40 at this site is running on a minicomputer (TI 990-12), and with this hardware, the absolute limit on trending is approximately 200 points. 


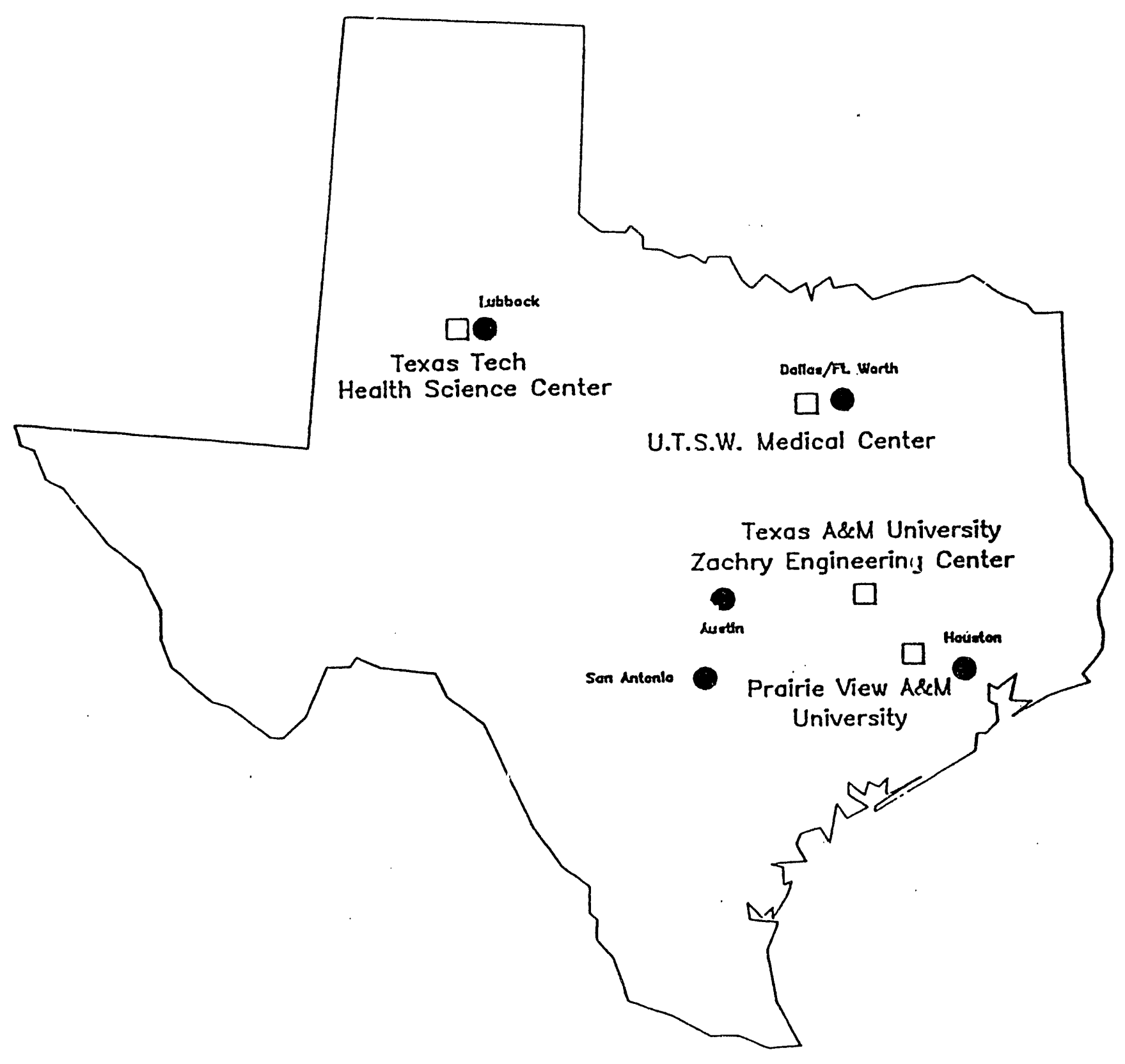

FIGURE 2-EMCS Case Study Sites. The locations of the case studies described in this report. Open squares indicate the sites, and filled circles indicate major cities for reference. 


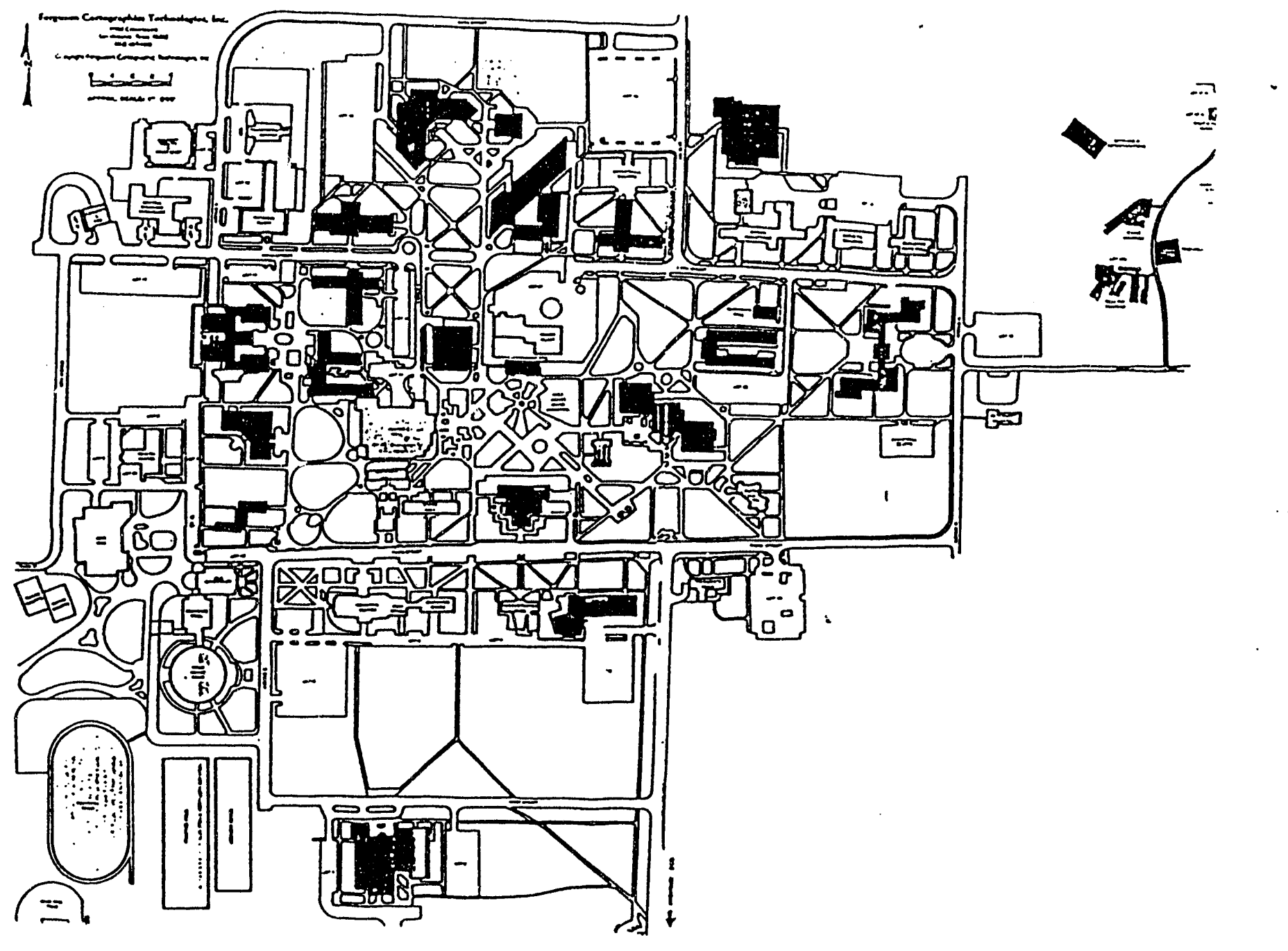

FIGURE 3-Prairie View A\&M University. The Prairie View A\&M University Campus, is located near Houston, and consists of about 46 buildings. 
TABLE 3-Typical Data From Prairie View EMCS.

For each Air Handling Unit:

\begin{tabular}{|c|c|c|c|c|c|}
\hline Device & Temp. & Stat.Press & RH & Setpt. & Status \\
\hline Cold Deck & - & & & $\bullet$ & \\
\hline Hot Deck & $\bullet$ & & & $\bullet$ & \\
\hline Reheat & - & & & $\bullet$ & \\
\hline Preheat & - & & & $\bullet$ & \\
\hline Supply Air & $\bullet$ & & $\bullet$ & - & \\
\hline Return Air Duct & $\bullet$ & & & $\bullet$ & \\
\hline Discharge & - & & & $\bullet$ & \\
\hline Fan & & $\bullet$ & & $\bullet$ & $\bullet$ \\
\hline Individual Zone(s) & $\bullet$ & & & $\bullet$ & \\
\hline
\end{tabular}

For Whole Building:

\begin{tabular}{|l|cccccc|}
\hline Device & Temp. & Flow & Energy & RH & Setpt. & Status \\
\hline Electrical & & & $\bullet$ & & & \\
CW Supply & $\bullet$ & $\bullet$ & $\bullet$ & & & \\
CW Return & $\bullet$ & $\bullet$ & & & $\bullet$ & \\
CW Pump & & & & & & $\bullet$ \\
HW Supply & $\bullet$ & $\bullet$ & $\bullet$ & & $\bullet$ & \\
HW Return & $\bullet$ & & & & & \\
HW Pump & & & & & & $\bullet$ \\
DHW Supply & $\bullet$ & & & & & \\
Condensate Tank & & & & & & $\bullet$ \\
Outside Air & $\bullet$ & & & $\bullet$ & & \\
\hline
\end{tabular}

There is also a facility for trending data at the level of the DSC, (Digital System Controller, or remote control unit). Up to 400 points can be trended at this level. For each point, only 64 samples can be stored at the DSC before new data begin to overwrite the oldest data. Data must therefore be uploaded to the $\mathrm{JC} / 85 / 40$ periodically. For example, if hourly data are collected at the DSC, they must be uploaded once every 64 hours, or about every other day. This can be done automatically by defining a weekly schedule.

Although the maximum number of trended points is limited by the software to about 600 , the actual number that can be trended may be limited by the communications rates. If too many points are trended, or points are sampled too frequently, cominunications within the system can become clogged, and system operation can be affected. According to the operators, communications paths in this system are already fairly loaded, and it would not be possible to trend many more points. The building operators currently use 35-40 trend points for facility operation. For troubleshooting, they sometimes use up to 80 points. It was therefore possible for us to use a few of the existing trend points. 
At the time of this study, Prairie View had not yet applied for a loan. Therefore, during this project, we did not know which buildings would receive retrofits. As a demonstration of the monitoring procedure, we have begun trending points representative of the kinds of points that are monitored in the LoanSTAR program. We selected to monitor the J.B. Coleman Library-the largest energy consumer on the campus. The points we began trending were:

- whole-building electrical power for Coleman Library;

- whole-building cooling power, for Coleman Library;

- whole-building heating power, for Coleman Library;

- outdoor air drybulb temperature; and

- outdoor air wetbulb temperature.

The energy data represented in the trend report are hourly samples of power, not hourly averages. The electrical power is measured using a pulse counter; each pulse represents a given amount of energy. The sample, then, represents the number of pulses recorded in the previous minute. The EMCS also has a facility for either totalizing or averaging values. With totalization, values such as energy nsumption are summed up to prepare monthly summaries. Averaging is used for variables such as temperatures too prepare monthly summaries. However, it is not possible to integrate totalization with trending, to trend hourly averages.

When trends are initialized, one selects the time for trending to begin, so that it is possible to collect data on the hour. These data are collected at each sample interval and stored on the JC/85/40. If the JC/85/40 loses power and restarts, it resumes trending normally, at the correct times. Of course it would lose the samples that should have come in while the system was down.

\section{Data Access}

After some initial logistical problems, we can now log on directly to the JC/85/40-the EMCS's central processor.

Although the trending facility is fairly sophisticated, it is not presently written to allow remote access to data. Trend data can be stored in a disk file, or printed to a printer. However, the disk file cannot be automatically transferred to our computer, and the trend data cannot be displayed on the screen to be subsequently captured into a $\log$ file by our computer. A solution to this limitation was found by personnel at the regional office of Johnson Controls. They set up the system so that remote dial-in computers are configured to be printers. In this way, when the JC/85/40 thinks it is printing data to a printer, it is actually going over phone lines, and appearing on our computer screen, and being saved in our log file. Figure 4 shows an example of the log file containing an entire EMCS session, including trend data. Figure 5 shows some of the data that were collected from this site.

There are several advantages to this method of remote access. Since very little input is required of the operator to request the trend data (only the login, and one command line for each point to be trended), this method lends itself very well to automation. Also, the trend report for a particular point includes the time and date of the first sample, the sample interval, and then a stream of data samples, ten per line (see again Figure 4). Therefore, since each sample does not take up a whole line, and is not individually time- 


\section{LOG-IN \& XXXX}

0, SUM, TRD, VAL, 43

O, SUR, TRD, VRL, A3, HCA

EXE/IGN (E/I) E (RET)

SYSTEM INFORMATION - TREND VALUE SUMAMRY PGM DAY 5 THU 8 AUG 1991 12:11:02 PAGE: 1

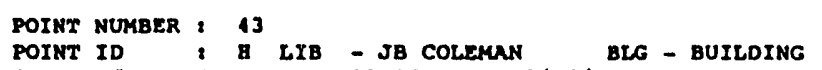

SAMPLE * BEGIN TIME, 11:00 ON 0/05/91

\begin{tabular}{|c|c|c|c|c|c|c|c|c|c|c|c|}
\hline 1. & 10 & 213.20 & 188.30 & 213.50 & OFFLINE & 186.90 & 191.20 & 200.50 & 182.90 & 213.80 & 180.60 \\
\hline $11-$ & 20 & 164.80 & 165.10 & 0.00 & 0.00 & 0.00 & 0.00 & 0.00 & 0.00 & 0.00 & 0.00 \\
\hline 21- & 30 & $\$ 18.00$ & OFFLINE & 172.00 & 184.00 & 190.10 & 188.70 & 193.40 & 150.80 & 179.40 & 164.10 \\
\hline $31-$ & 10 & 161.80 & 169.30 & 181.70 & 146.40 & 182.70 & 196.20 & 0.00 & 0.00 & 0.00 & 0.00 \\
\hline 11- & so & 0.00 & 0.00 & 0.00 & 0.00 & 254.10 & 177.50 & 174.00 & 172.30 & 171.50 & 168.80 \\
\hline 51- & 60 & 176.80 & 181.90 & 162.20 & 166.70 & 180.20 & 166.70 & 181.70 & 143.00 & 158.70 & 157.60 \\
\hline $\begin{array}{l}61- \\
71-\end{array}$ & $\begin{array}{l}70 \\
80\end{array}$ & $\begin{array}{r}0.00 \\
159.00\end{array}$ & $\begin{array}{r}0.00 \\
145.20\end{array}$ & $\begin{array}{r}0.00 \\
154.60\end{array}$ & $\begin{array}{r}0.00 \\
172.20\end{array}$ & 0.00 & 0.00 & 0.00 & 0.00 & 262.10 & 132.50 \\
\hline
\end{tabular}

Qن̈IT...

FIGURE 4-Log file from Prairie View A\&M EMCS. In an EMCS session, trenc data are displayed to the screen, and the entire session is captured to this file. User responses are indicated in boldface. 

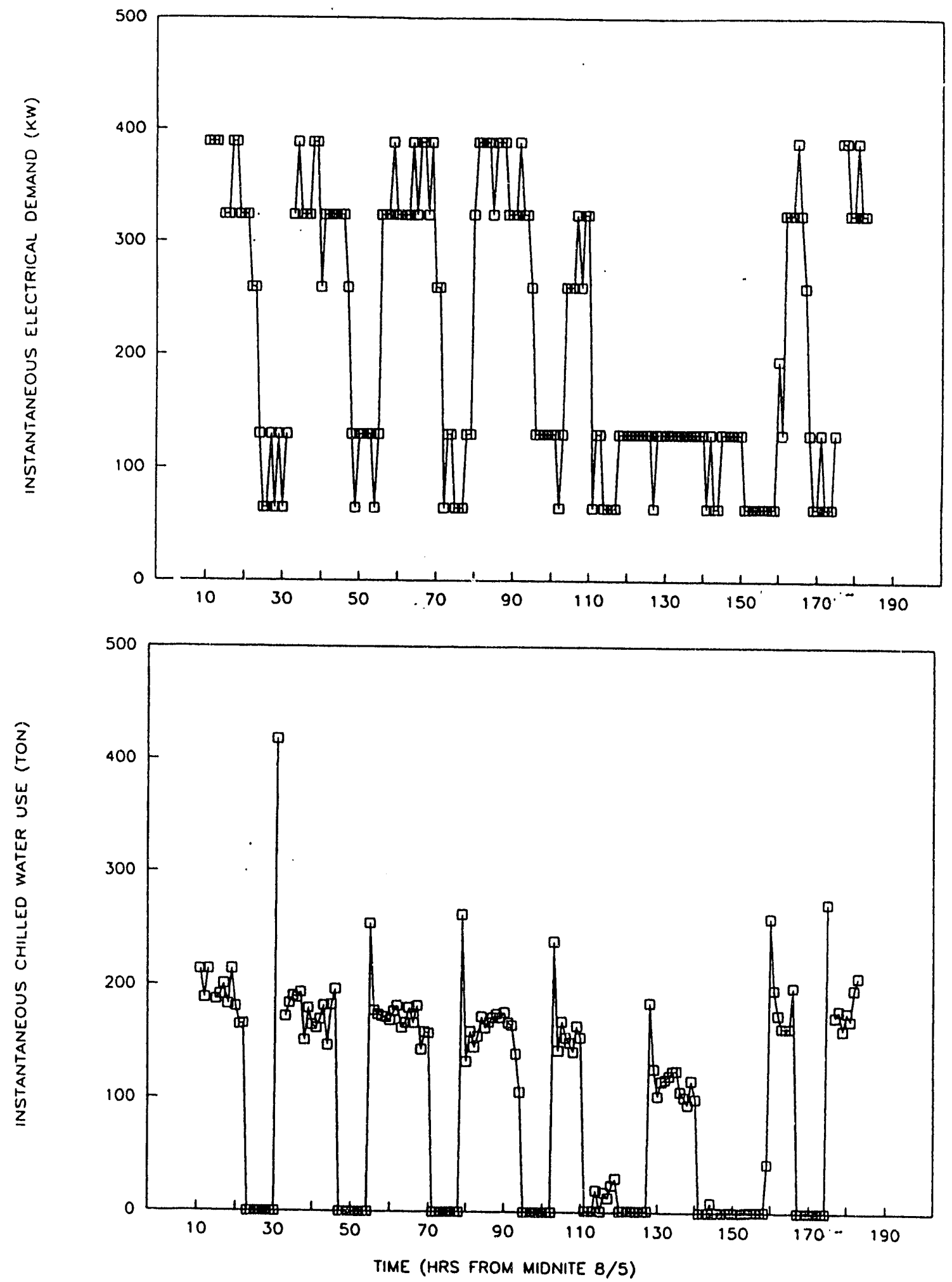

FIGURE 5-Prairie View A\&M EMCS Data. This is a sample of the data retrieved from the EMCS at Prairie View A\&M. Hourly samples of instantaneous demand (a) and chilled water use (b) are shown for about a week, for one building. The demand data illustrate the need for hourly total energy measurements, rather than instantaneous demand. 


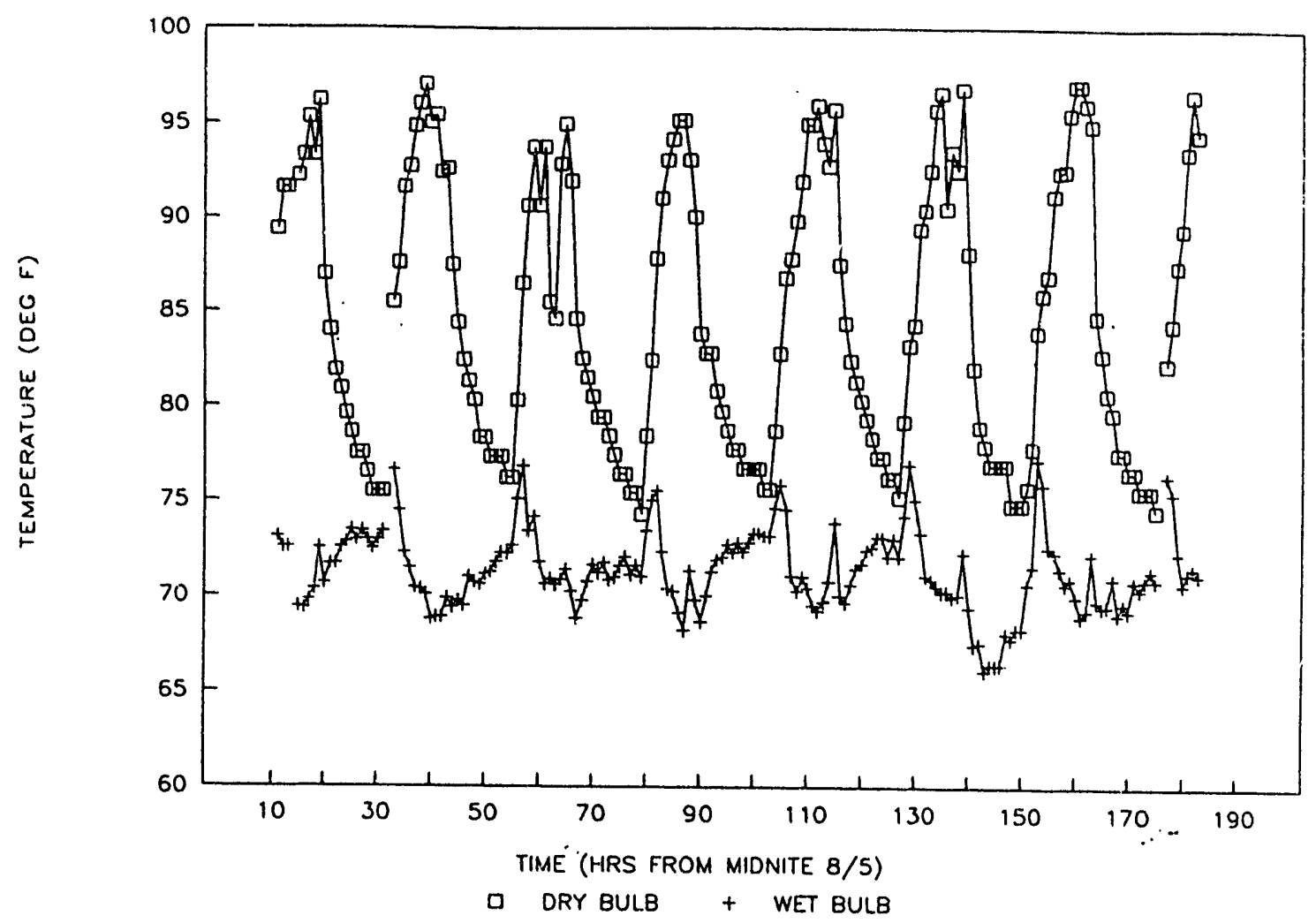

FIGURE 5 (cont.)-Prairie View A\&M EMCS Data. Instantaneous outdoor drybulb and wetbulb temperature data collected from the EMCS at Prairie View A\&M. 
stamped, data transmission time is minimized.

However, there are also several problems with this method of remote data access. First, it is not possible to check for data transmission errors during polling. Secondly, although no manufacturer software modifications were necessary, the system had to be site-reconfigured. The site personnel did not have the expertise to do this. It was also a problem because once the system is reconfigured to recognize dial-ins as printers, all dial-ins were recognized as printers, including operators calling in from home to check on the system, or the regional office calling in to trouble shoot problems. Apparently, this is not a severe problem, although the interface is less user-friendly: the user must enter all commands on a command line and is not prompted with a menu.

We also considered an alternate method of remotely accessing the data, using a PC-based Extended Application Workstation (EAW). An EAW is a personal computer containing a Johnson Controls network card, and running EAW software. It communicates over an RS-232 connection to the JC/85/40. The monitoring project could purchase a PC to serve as such a workstation. This PC would reside onsite, and it would periodically run the data base manager program to collect trend data from the JC/85/40. The data base manager has facilities to periodically upload trend data from the $\mathrm{JC} / 85 / 40$, and to translate these data into an ASCII DOS file in "DIF" format. "DIF" stands for Data Interchange Format, and was developed for Visicalc, an early microcomputer spreadsheet program. DIF is a file format that can be written and read by different programs. Although it is a very bulky way of transmitting data, the format is quite suitable for LoanSTAR purposes. We could then dial into the EAW, and transfer this file.

Unfortunately, purchasing a PC to serve as an EAW is a relatively expensive solution to the problem. It may be possible to make use of one of the other existing EAWs. Limitations on this approach are:

- space on the hard drive of the existing computer-we were told that the hard drive on at least one of the installation's EAWs was already quite full, and probably would not have space for our data;

- sensitivity of the operation of the EAW—one of the EAWs at this site is dedicated to fire and security operations, and they would not allow us to use it for a relatively low priority task such as data collection;

- capabilities of the existing EAW-the current EAW resides on an older 286-class computer and is somewhat slow.

Another way to reduce the cost of using a PC as an EAW would be if the EAW could be located in a remote location, such as in the LoanSTAR office. Since the connection between the EAW and the $\mathrm{JC} / 85 / 40$ is an RS-232 cable, it may be possible that a modem and phone-line could be included in the connection. It would theoretically be possible to have one computer dedicated to downloading data from several Johnson Controls sites, using the Johnson Controls hardware and software, or possibly to have hardware and software from several different manufacturers contacting different sites. We would also need to make sure that the EAW could support an Ethernet adapter or other means of connecting this computer to the LoanSTAR network. 


\section{Data Processing}

Processing of the data is fairly straightforward for this site. The log file includes a command line, a header, and lines of data samples. The headers must be removed, and a time stanip for each sample must be calculated. One additional processing step is that the convention in the LoanSTAR database is to have one row of data for each time period, consisting of several different data points. Therefore, several different points must be placed next to each other to form columns of data. All of these processing steps can be done in AWK.

\section{CASE STUDY 2: TEXAS TECH UNIVERSITY HEALTH SCIENCE CENTEIR}

\section{Building and EMCS}

The Texas Tech University Health Sciences Center (TTHSC) is a very large medical research building located in Lubbock (see Figure 6). The 811,000 square foot facility is scheduled to receive conservation measures including lighting retrofits, variable-speed air handler conversion, HVAC economizers, night setback controls, steam trap repair, pipe insulation, and new heating and chilled water pump controls. Additional conservation measures planned for the heating and cooling plant serving the facility include chiller replacement, variable speed chilled water pumping, and steam turbine electrical generation at a pressure-reducing station. Expected savings from the measures total $\$ 920,000 /$ year, with an overall simple payback time of 3.4 years.

The TTHSC is equipped with a Honeywell Delta 1000 EMCS with approximately 1800-2000 points (see Table 4). The system is instrumented for energy monitoring at each of the four mechanical "pods" serving the building. These points include chilled water supply and return temperatures and flow, condensate flow, and electrical consumption for each pod. Weather sensors and condensate meter points were added under the LoanSTAR monitoring funds. EMCS operators at TTHSC have been visited by LoanSTAR staff and have shown a favorable interest in the project.

\section{Data Storage}

The Energy Auditor software module was purchased and installed to allow trending at this site. The Energy Auditor program allows trending of up to 999 points, so trending capacity is not a problem at this site. The Director of Plant Operations Engineering at Texas Tech set up data trends for the following points:

- $\quad$ electrical power, for each pod;

- cooling energy, for each pod;

- $\quad$ steam flowrate, for each pod;

- chilled water flowrate, for each pod;

- $\quad$ chilled water load, for each pod;

- chilled water supply temperature, for each pod; 


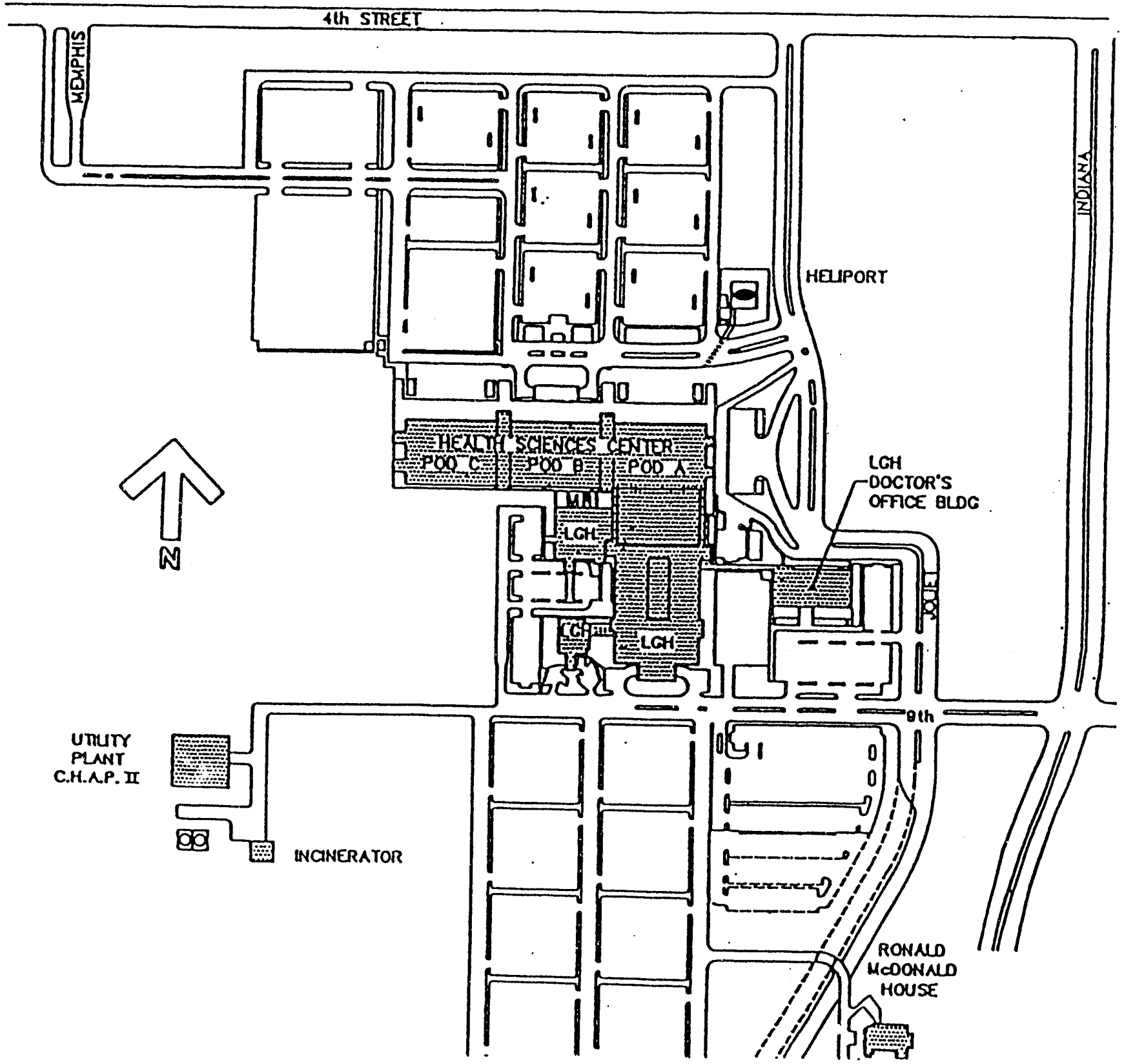

FIGURE 6-Texas Tech Health Sciences Center. The Texas Tech Health Sciences Center is a 811,000 square foot facility located in Lubbock. It is split into four mechanical "pods." 
TABLE 4-Typical Data From Texas Tech EMCS.

For each Air Handling Unit:

\begin{tabular}{|c|c|c|c|c|c|}
\hline Device & Temp. & Stat.Press & RH & Setpt. & Status \\
\hline Cold Deck & $\bullet$ & & & • & \\
\hline Hot Deck & $\bullet$ & & & $\bullet$ & \\
\hline Reheat & - & & & - & \\
\hline Preheat & $\bullet$ & & & $\bullet$ & \\
\hline Supply Air & $\bullet$ & & $\bullet$ & - & \\
\hline Return Air & - & & & $\bullet$ & \\
\hline Discharge & $\bullet$ & & & $\bullet$ & \\
\hline Fan & & • & & - & $\bullet$ \\
\hline Individual Zone(s) & $\bullet$ & & & $\bullet$ & \\
\hline
\end{tabular}

For Whole Building:

\begin{tabular}{|l|cccccc|}
\hline Device & Temp. & Flow & Energy & RH & Setpt. & Status \\
\hline Electrical & & & $\bullet$ & & & \\
CW Supply & $\bullet$ & $\bullet$ & $\bullet$ & & & \\
CW Return & $\bullet$ & $\bullet$ & & & $\bullet$ & \\
CW Pump & & & & & & $\bullet$ \\
HW Supply & $\bullet$ & $\bullet$ & $\bullet$ & & $\bullet$ & \\
HW Return & $\bullet$ & & & & & $\bullet$ \\
HW Pump & & & & & & $\bullet$ \\
DHW Supply & $\bullet$ & & & & & \\
Condensate Tank & & & & & & $\bullet$ \\
Outside Air & $\bullet$ & $\bullet$ & $\bullet$ & $\bullet$ & & \\
\hline
\end{tabular}

- $\quad$ chilled water return temperature, for each pod;

- condensate return flowrate, for each pod;

- wind speed;

- solar insolation;

- outdoor air drybulb temperature; and

- outdoor air humidity.

Data collection begins as soon as the trend puints are initialized, which may or may not fall on the hour. If the system is ever powered down and rebooted, data collection begins immediately. Therefore, it is impossible to ensure that hourly data values will be recorded at the top of the hour. Additionally, most of the data values are instantaneous samples, rather than hourly averages, which is particularly a problem for 
the energy points.

\section{Data Access}

We have successfully dialed into the Honeywell EMCS at Texas Tech, and run the Energy Auditor (see the EMCS log in Figure 7). Unfortunately, the Energy Auditor does not allow remote access to the trended data. Trended data can be stored to a disk file, but that file cannot be automatically transferred to our computer. The data can be printed to a printer, but not to the screen.

The only way we were able to access the data was to have the data stored to disk files, and have the Texas Tech EMCS operators send diskettes of trended data to A\&M, via the mail, (see data in Figure 8). This manual mailing method proved to be slow, and we arranged to have the data sent electronically via FTP, the ARPANET standard File Transfer Protocol. To do this, the data are transferred from the PC-based computer to a mainframe running UNIX. The data are then sent over a computer network at a very high transmission rate and with very high reliability (error checking and correction). This method gives us the data we need for now, but a more automated method will have to be developed, that does not rely on operator assistance. We will try to work with Texas Tech and Honeywell personnel to develop another way of accessing data. Discussions with Honeywell indicate that they may be interested in some form of cooperative work on developing this type of procedure. According to the director of Plant Operations Engineering, Honeywell has identified TTHSC as a test location for developing the software needed to do remote accessing.

\section{Dala Processing}

Once the data files have been delivered (either manually or electronically) to LoanSTAR, they are fairly simple to process. The files are stored in DIF format, and Microsoft Windows ${ }^{2}$ was used to translate from DIF format to text. Once in text format, headers and unnecessary information are easily removed using AWK. Figure 9 shows the data processing stream for these data. Some points are reported as accumulating values. One step in processing, then, involves subtracting one value from the next to calculate the hourly change.

\section{CASE STUDY 3: UNIVERSITY OF TEXAS SOUTHWEST MEDICAL CENTER}

\section{Building and EMCS}

The University of Texas Southwest Medical Center (UTSMC) is a large medical research center located in Dallas. A map of the facility is shown in Figure 10. The campus received an energy audit in 1986. Several retrofits were recommended in buildings $F, G, H$, and $J$, so these buildings were the focus of our earliest monitoring efforts. As LoanSTAR negotiations continued, other buildings were identified as more likely retrofit candidates. Finally, however, it was determined that this site would not be a LoanSTAR loan recipient, and further monitoring efforts were suspended. Although this site will not be part

2 Microsoft Windows is a registered trademark of the Microsoft Corporation. 


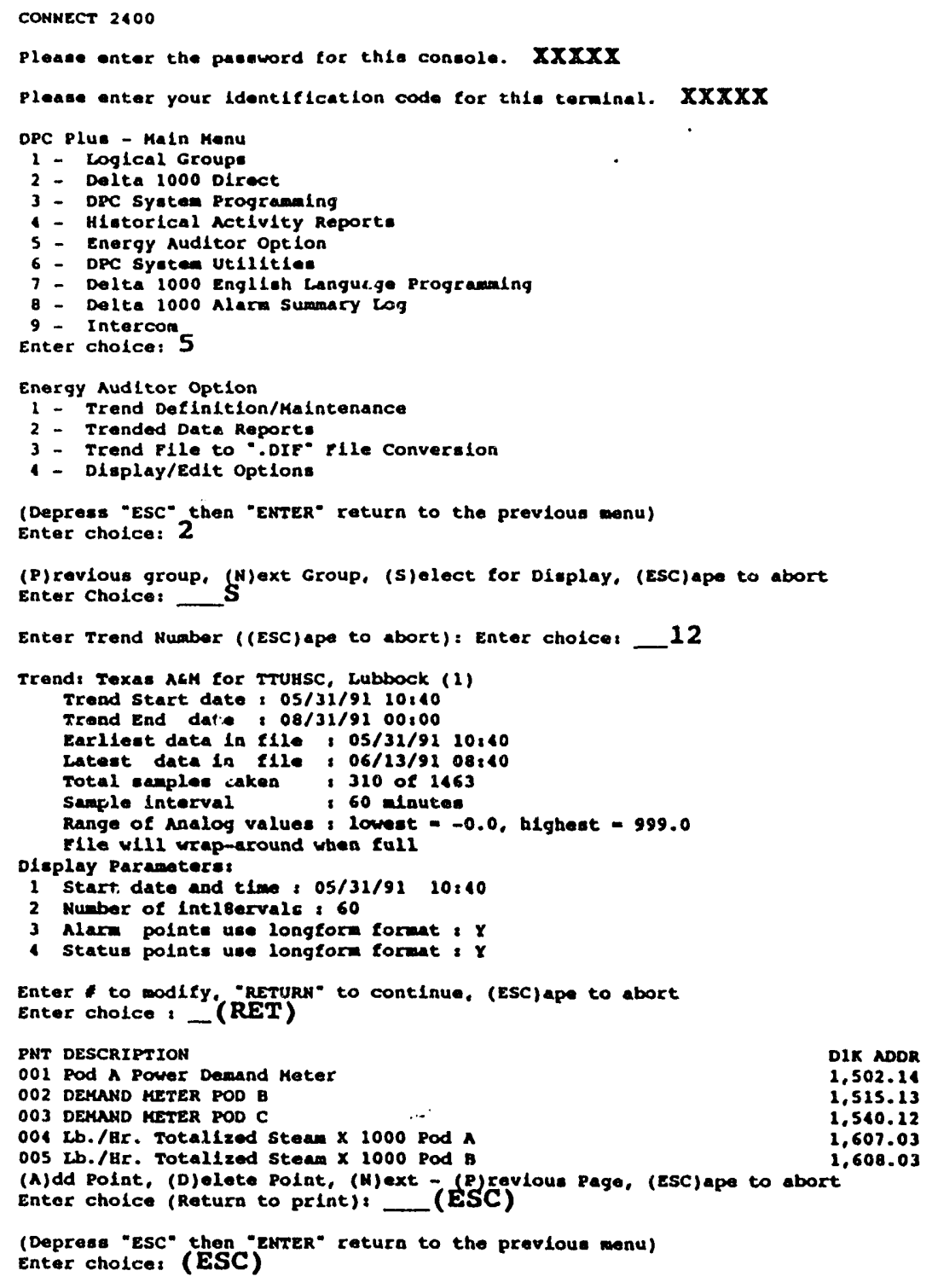

FIGURE 7-Log file from Texas Tech EMCS. This log documents an EMCS session using the Energy Auditor software. It was not possible to display data to the screen. 

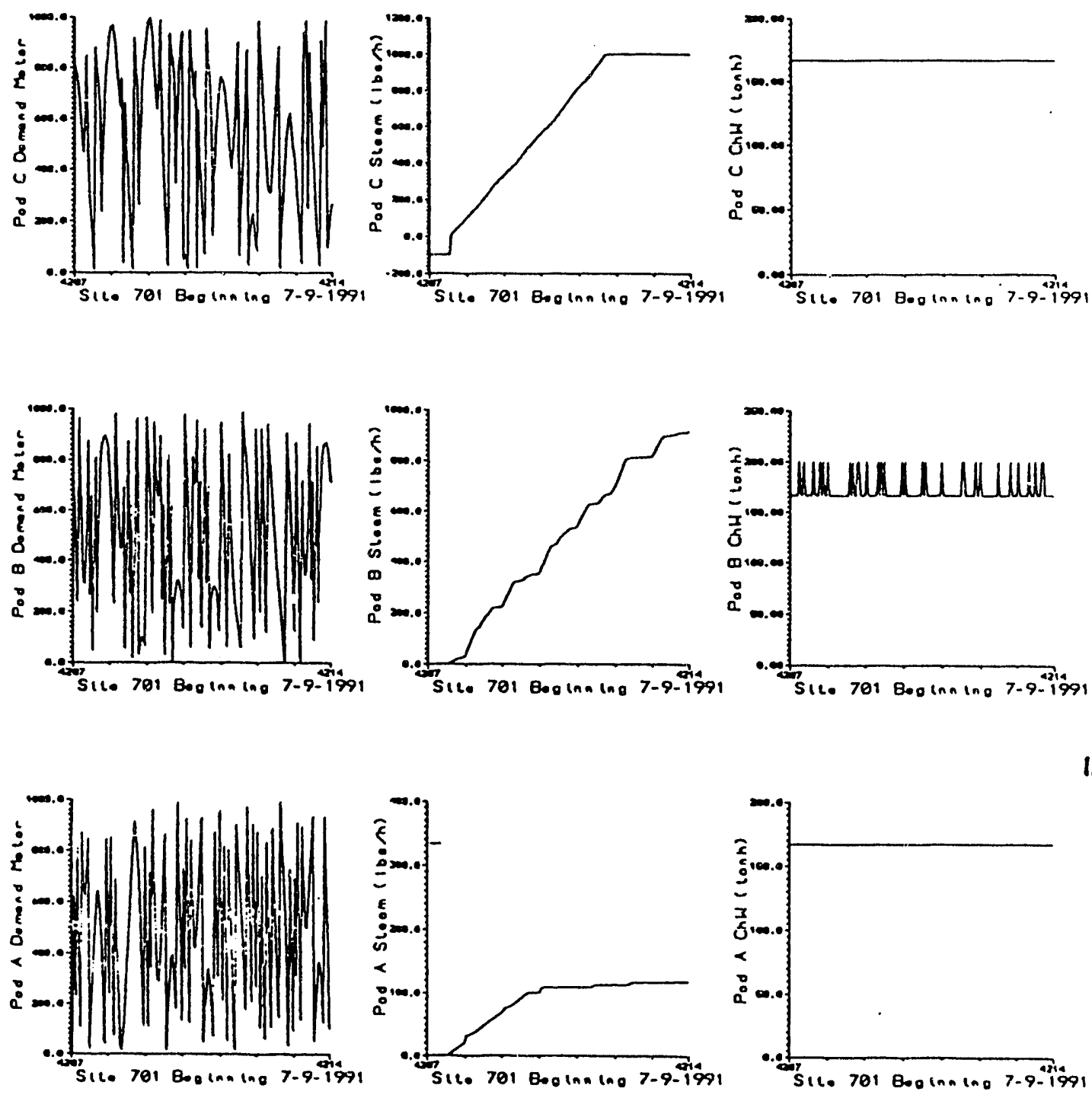

Texas A\&M University Energy Systems Laboratory INTERNAL DISTRIBUTION

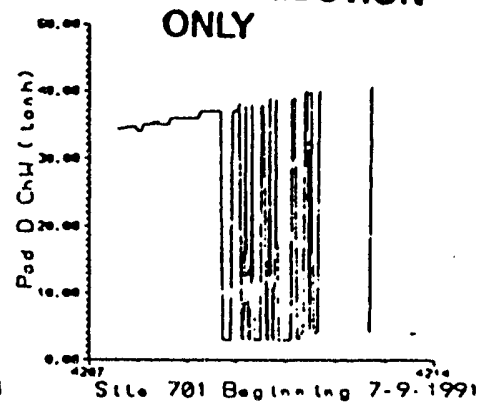

FIGURE 8-Texas Tech EMCS Data. This figure contains an example of the inspection plots that are produced each week when the Texas Tech data are retrieved from the site. Note that the data shown represent early attempts to obtain data from the site. The demand data are instantaneous values, and therefore appear quite erratic. The steam values are cumulative, and differences should be calculated. 


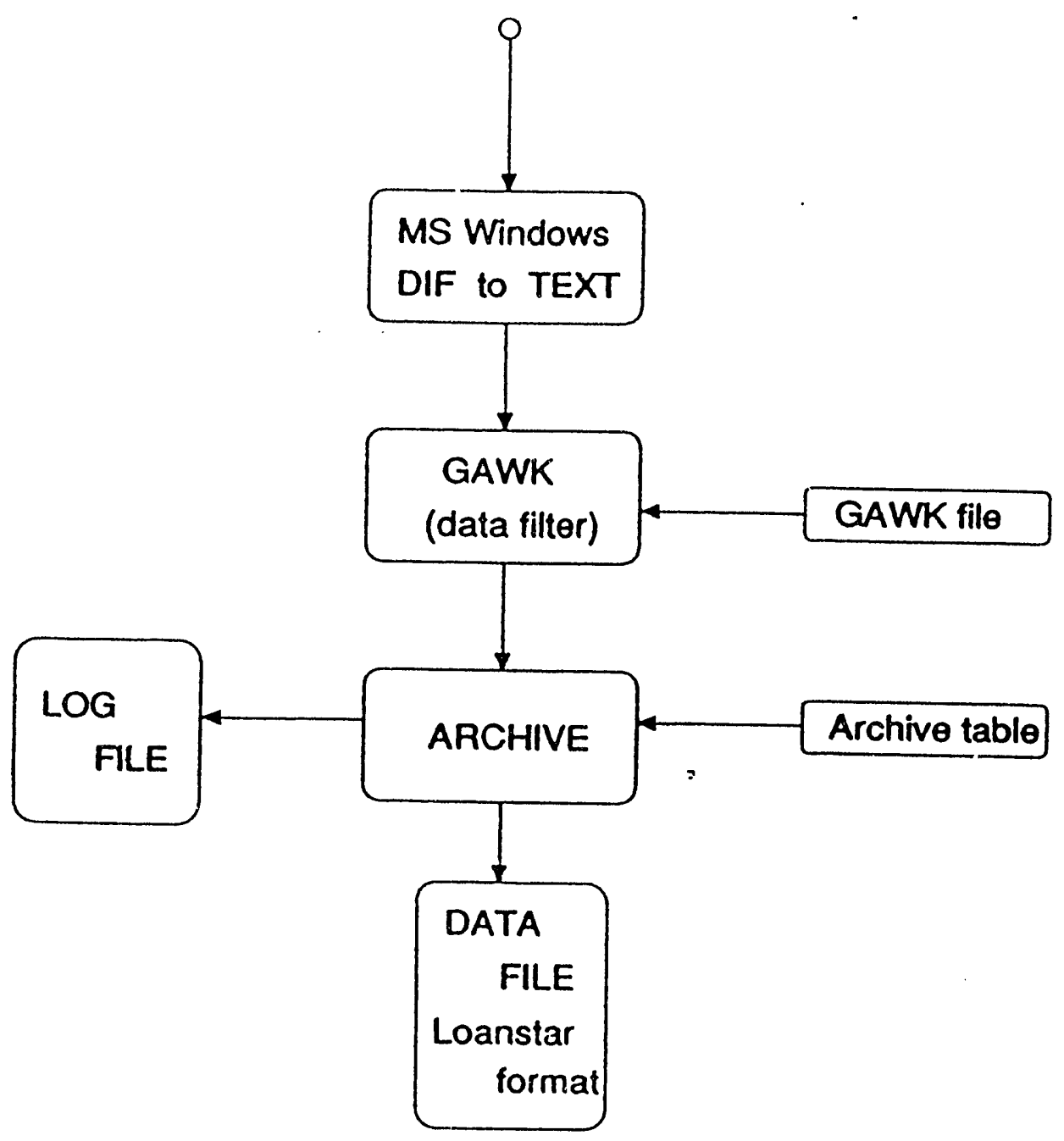

FIGURE 9-Texas Tech Data Processing Stream. The data from this EMCS are converted to DIF format by the Energy Auditor Software. After the data are received by LoanSTAR, they are converted to text, filtered, and archived. 
of the LoanSTAR program in the future, it is included in this report because our experience with monitoring there was quite valuable.

The campus is equipped with a Landis \& Gyr, Powers System 600 EMCS with nearly 6000 connected points. The types of points connected to the system for each air handling unit and for the whole building are summarized in Table 5. One should note that building $F$ has nine AHUs, $G$ has four, $H$ has ten, and $J$ has eight, so a large volume of data is available. All of the buildings are on a central utility electricity meter, and steam and chilled water are circulated to each building from a central plant.

Each of the four buildings we were initially interested in has one or two electrical meters. A pulse generator is installed on each meter, and generates a pulse each time a given amount of energy is consumed. The pulses are counted and collected by the EMCS. The energy consumption is saved as cumulative $\mathrm{kWh}$, so one reading must be subtracted from the next to determine incremental energy consumption. The reading is supposed to be zeroed at the beginning of every month, but this appears not to be done regularly. The electrical meters were installed 18 years ago and have never received maintenance. The audit of the facilities recommended recalibration of these meters.

Unfortunately, no HVAC water flow data are collected. The supply and return temperatures of the chilled water and the steam condensate temperature are measured, but flowrate data are needed in order to calculate thermal energy consumption of the buildings. Turbire flowmeters are installed in almost all building condensate and chilled water supply lines, but they are nor providing reliable data, and hence they are not connected to the control system, although EMCS data channels have been allocated for these meters. If functioning meters were installed, they could easily be incorporated into the EMCS system. This was also recommended in the audit of the facility.

\section{Data Storage}

This Landis \& Gyr, Powers EMCS uses an interesting methoo of data collection. A system can either collect conventional hourly data, or use a technique known as Change of Value (COV) to record information. The system at this site uses COV trending. In COV trending, the system collects a piece of data only when its value changes. Since an EMCS is capable of a high degree of accuracy in measuring point values, it would greatly overload the flow of data across the network trunk lines if data were collected every time the last decimal place changes. Therefore, a factor must be defined for each point, the COV, which determines how much a value must change before it is considered a new value. For example, for a temperature point, if the COV is defined as one degree, nothing will be recorded until the temperature has changed by one degree. This clearly has several advantages. If a value is not changing at all, it would be wasteful to continually record its value. Conversely, if a value is changing rapidly, it is of much greater interest, and short time interval data would be valuable.

COV-based data have made analysis of the electrical consumption in the building more difficult. The electrical meters in the four buildings have been set for a COV of $300 \mathrm{kWh}$. Data are recorded whenever $300 \mathrm{kWh}$ have gone by, so the data are always in $300 \mathrm{kWh}$ increments. However, it also records the time when the consumption passed $300 \mathrm{kWh}$, so this information can be used to create a profile similar to an hourly profile. Since this method of collecting data is slightly different from the hourly method more familiar to energy analysts, it is worth a more thorough discussion, as found in the Appendix. 


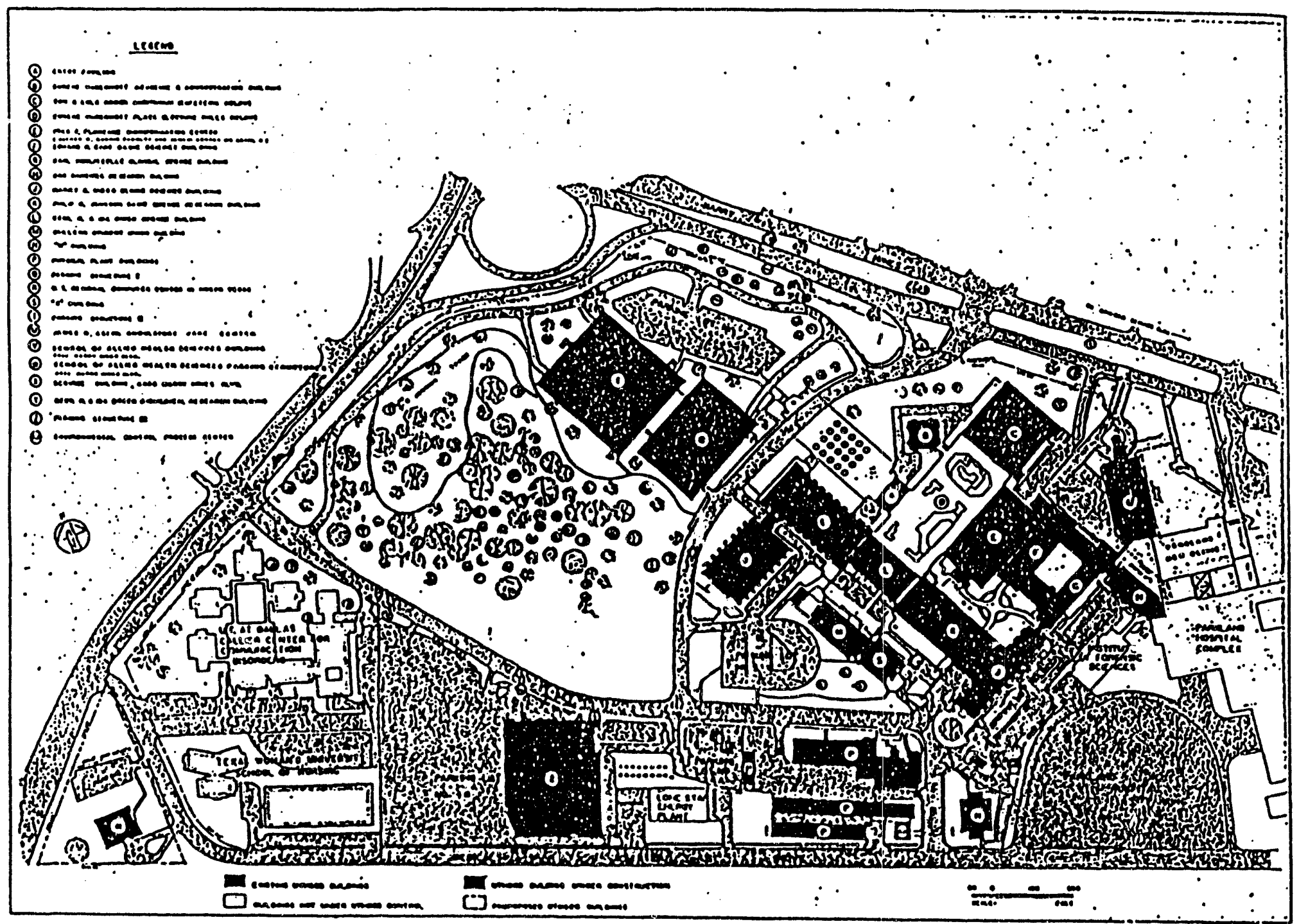

FIGURE 10-University of Texas Southwestern Medical Center. UTSMC is located in Dallas, and consists of about 23 buildings. 
TABLE 5-Typical Data From UTSMC EMCS.

For each Air Handling Unit:

\begin{tabular}{|c|c|c|c|c|}
\hline Device & Temp. $\quad$ RH & Position & Setpt. & Status \\
\hline Cold Deck & $\bullet$ & Valve & $\bullet$ & \\
\hline Hot Deck & $\bullet$ & Valve & $\bullet$ & \\
\hline Precool & $\bullet$ & Valve & $\bullet$ & \\
\hline Preheat & $\bullet$ & Valve & - & \\
\hline Preheat Supply & - & & & \\
\hline Preheat Return & $\bullet$ & & & \\
\hline Supply Air & $\bullet$ & Damper & $\bullet$ & \\
\hline Return Air & $\bullet$ & Damper & $\bullet$ & \\
\hline Mixed Air & $\bullet$ & Damper & - & \\
\hline Supply Fan & & & & - \\
\hline Return Fan & & & & $\bullet$ \\
\hline Individual Zone(s) & $\bullet$ & Damper & $\bullet$ & \\
\hline
\end{tabular}

For Whole Building:

\begin{tabular}{|c|c|c|c|c|c|c|}
\hline Device & Temp & Energy & RH & Position & Setpt. & Status \\
\hline Electrical & & - & & & & \\
\hline CW Primary Supply & $\bullet$ & & & Valve & & \\
\hline CW Primary Return & - & & & & & \\
\hline CW Secondary Supply & $\bullet$ & & & & - & \\
\hline CW Secondary Return & - & & & & & \\
\hline CW Differential & $\bullet$ & & & & & \\
\hline CW Pump & & & & & & - \\
\hline HW Supply & - & & & Valve & - & \\
\hline HW Pump & & & & & & - \\
\hline Reheat Supply & $\bullet$ & & & Valve & $\bullet$ & \\
\hline Reheat Return & $\bullet$ & & & & & \\
\hline Reheat Pump & & & & & & $\bullet$ \\
\hline DHW Supply & $\bullet$ & & & & & \\
\hline DHW Return & $\bullet$ & & & & & \\
\hline Condensate Return & • & & & & & \\
\hline Exhaust Fan(s) & & & & & & $\bullet$ \\
\hline Outside Air & - & & - & & & \\
\hline
\end{tabular}

The EMCS at UTSMC has three facilities to record and report data: trending, remote trending, and archiving. The trending facility is capable of recording data for 50 variables: five groups at ten points each. These data are originally acquired by the remote control unit, but then are immediately transmitted over the network to be stored on the host computer's disk. Few of the available trend points are being 
used at the site, so trend capacity is not a problem.

Remote trending is a facility very similar to trending, except that the data are stored in the local memory of a remote control unit, and are only transferred to the host computer upon request. With remote trending, the limitation on the amount of data that can be collected comes from the amount of memory available on the remote unit.

In the archive facility, the data are transmitted just as in the trending facility, and are then stored in a dynamic file, to be archived to a more permanent disk file once a day. Only this permanent file can be accessed, so data within the last day are not available. At this facility, the archive file is stored to magnetic tape once every two weeks.

The points that were monitored for this project are:

- whole-building electrical energy, for each building, one or two meters per building;

- chilled water supply temperature, for buildings $\mathrm{G}$ and $\mathrm{H}$;

- $\quad$ chilled water return temperature, for buildings $\mathrm{G}$ and $\mathrm{H}$;

- chilled water temperature difference, for buildings $\mathrm{G}$ and $\mathrm{H}$; and

- $\quad$ steam condensate temperature, for buildings $F, G$, and $H$.

\section{Data Access}

Figure 11 shows the procedure for logging on, requesting an archive report, and logging off. The procedure for trend and remote trend reports are similar. Figure 11 also shows the format in which the archive data are retrieved. The processed data are shown in Figure 12.

There are several problems with this method of downloading data. First, the data reports are defined to fit on a 132-column display, but the EMCS is configured to display only 80 columns. Therefore, several columns are truncated and their data are not accessible. The second problem is that no error checking is possible when simply capturing characters as they are displayed on the screen. Finally, quite a bit of user interaction is required to request a report. This suggests that automation of the procedure would be difficult.

Two alternate methods for transferring the collected data may solve these problems. One is to obtain a copy of the magnetic tape on which the building operators store the archive data. This tape could be picked up periodically. The other alternative also makes use of the archive utility provision to save data in a spreadsheet-compatible file, and send the data file using the Kermit file transfer protocol. This would have the advantage of automatic error checking, and would also require no postprocessing (see below). It would have to be set up by the building personnel, however, and would require the purchase of an IBM PC compatible computer for the facility. 
MNE, ENERGX
PASSWORDS XXXX

\section{ARCE}

SYHTAX ACCEPTED

SYSTEM 600 NRCHJVE PACKAGE

MAIN RENU

1) POINT REPORTS

2> MESSAGE REPORTS

3) DATABASE MATHTENANCE

() ARCHIVE OPERATIONS

ENTER SELECTION $(1-4, Q, H=H E L P) \ldots, 1$

POINT REPORTS MENU

i> DETAILED VALUES

2) AVERAGE, HIGH, LOW VALUES

3> PERTODIC SNMPLES

1) LONG TERM

ENTER SELECTION $(1-4,0) \ldots: .1$

- detaILED poInt REPORT MENU -.

1) MULTIPLE POINTS, DATE NND TIKE RANGE

2) ONE POINT, MULTIPLE DATES, TIME RNHGE WITHIN DATE

3) MULTIPLE POINTS, MULTIPLE OATES, TIKE RANGE WITHIN DATE

ENTER SELECTION $(1-3,0) \ldots \ldots 2$

DESTINATION $(0, R, p, 0)$..... D

GROUP NAME, "CRET>" MONE: (RET).

DETAILEO POINT REPORT PORK...

POINT HAME, GEMLKW

BEGINWING TIME: OOO

ENDING TIME: 2400

DATE

1) OgJAN91

2) IOJAN9 I

3) $1 \frac{1}{2}$ JAN9 1

6)

8)

9)

hes

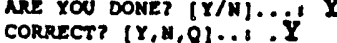

ARCHIVE REPORT INITIATED - COLLECTING DATA

\begin{tabular}{|c|c|c|c|c|}
\hline TIME & $\begin{array}{l}\operatorname{GEN} 2 K W \\
9-J N N-91\end{array}$ & $\begin{array}{c}\text { GEH2KN } \\
10-J A N-91\end{array}$ & $\begin{array}{c}\text { GEM2KH } \\
11-J A N-91\end{array}$ & $\begin{array}{c}\text { GEN2KW } \\
12-J A N-91\end{array}$ \\
\hline $\begin{array}{l}0: 50: 16 \\
1: 09169 \\
1115: 18 \\
2: 13: 08 \\
1: 18: 47 \\
1126: 12 \\
1: 38: 53 \\
5114154 \\
7: 26: 45\end{array}$ & 23329.5 & 34991.3 & $\begin{array}{c}38225.7 \\
38549.1\end{array}$ & $\begin{array}{l}11136.6 \\
11460.1\end{array}$ \\
\hline $\begin{array}{l}7: 38: 21 \\
7: 39: 54 \\
8: 21: 39 \\
9: 21: 12 \\
9: 28: 41 \\
10: 08: 00 \\
10: 30: 27 \\
10: 30: 27 \\
11: 01: 15\end{array}$ & 23977.5 & $\begin{array}{l}35314.8 \\
35638.2\end{array}$ & 38072.6 & $\begin{array}{r}12106.9 \\
12106.9\end{array}$ \\
\hline $\begin{array}{l}11: 01: 15 \\
11: 06: 11 \\
11: 16: 32 \\
11: 16: 32 \\
12: 11: 25 \\
13: 05: 01 \\
13: 26: 27 \\
11: 21: 11 \\
15: 03: 47\end{array}$ & 24625.6 & $\begin{array}{l}35961.6 \\
35962.6 \\
36285.1 \\
36608.5\end{array}$ & 39519.4 & 12430.4 \\
\hline $\begin{array}{l}15: 33: 20 \\
15: 33: 20 \\
15: 51: 03 \\
16: 04: 18 \\
16: 47: 03 \\
17: 20: 02 \\
10: 18: 37 \\
18: 46: 26 \\
29: 02: 47\end{array}$ & $\begin{array}{r}x \times x \times x \times x \times x \\
33375.2\end{array}$ & 36931.9 & 10166.3 & 43077.3 \\
\hline $\begin{array}{l}19: 42: 13 \\
21: 20: 36 \\
21: 56: 38 \\
22: 01: 33 \\
22: 46: 35\end{array}$ & 34344.4 & 37570.8 & 40813.2 & 43400.7 \\
\hline
\end{tabular}

ARCHIVE REPORT COMPLETED

BYCHE

FIGURE 11-Log file from UTSMC EMCS. In an EMCS session, archive data are displayed to the screen, and the entire session is captured to this file. User responses are indicated in boldface. 

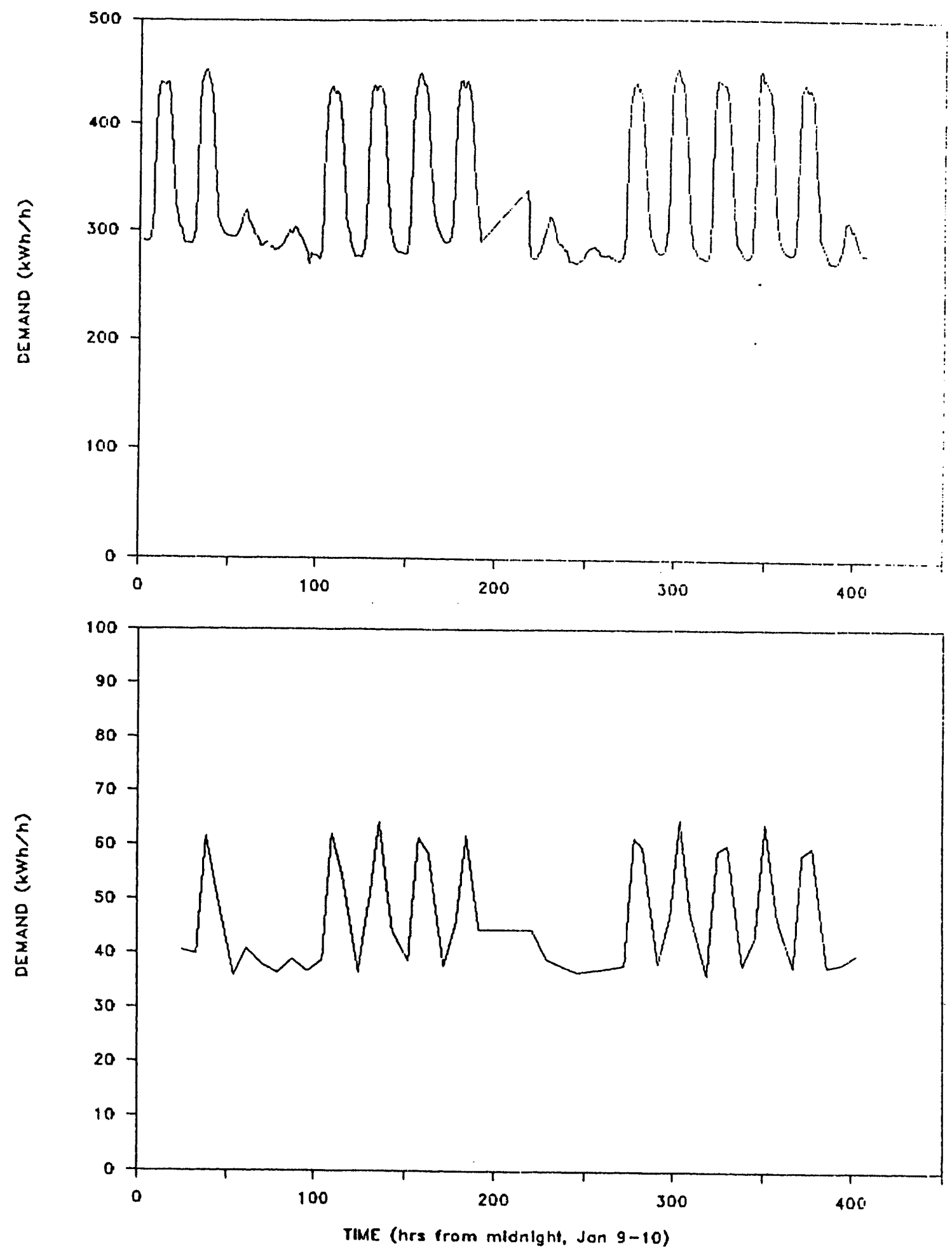

FIGURE 12-UTSMC EMCS Data. This is a sample of the data retrieved from the EMCS at UTSMC. Demand data in COV format for about a 17 days. The demand of building F (a) is greater than the COV rate $(300 \mathrm{kWh} / \mathrm{COV})$, so data are collected at a frequency greater than hourly. The demand for building $\mathrm{J}$ (b), however, is much lower, and hence data are recorded less frequently than once per hour. 


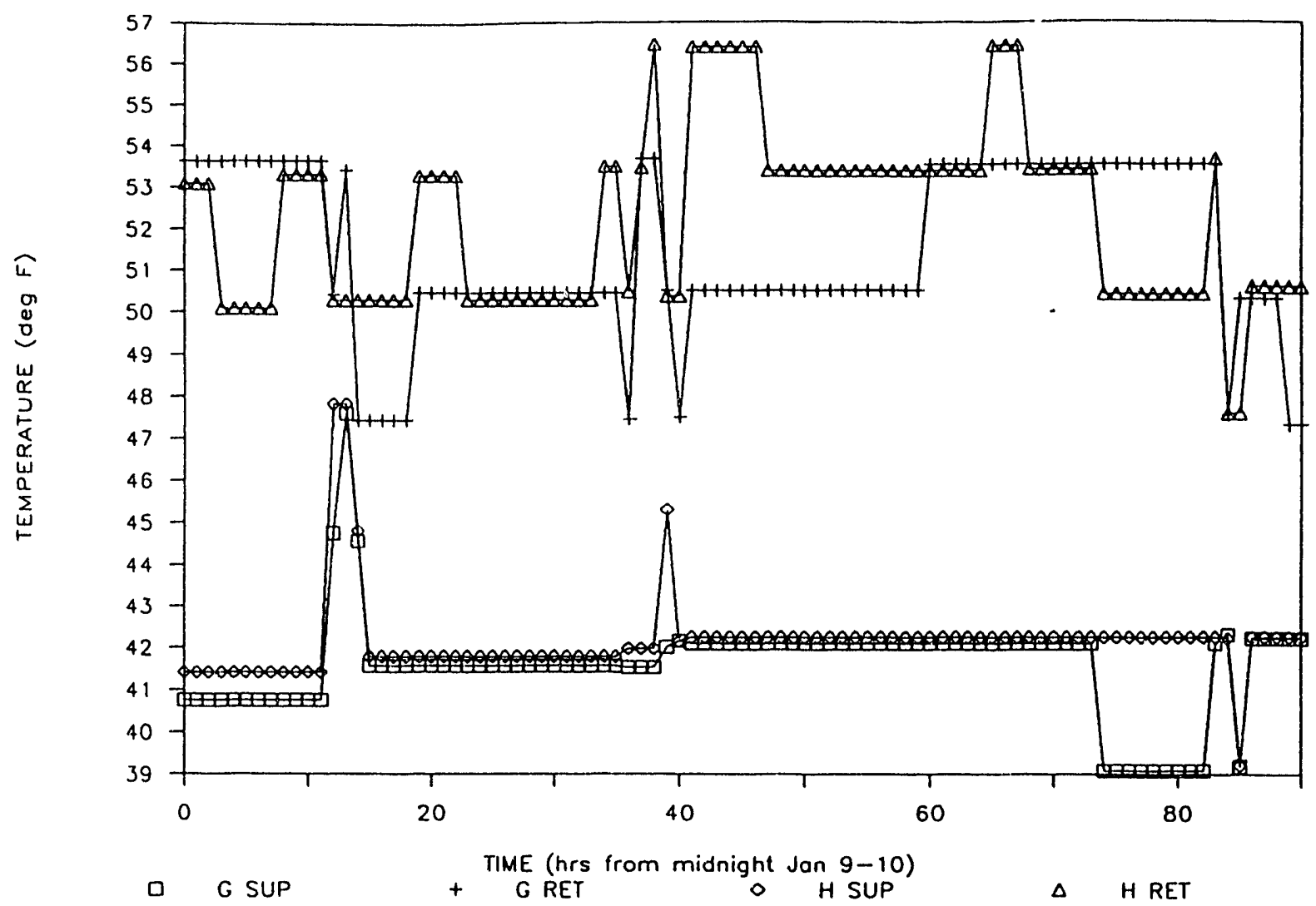

FIGURE 12 (cont.)-UTSMC EMCS Data. A sample of temperature data retrieved from the EMCS at UTSMC. The chilled water supply and return temperatures for buildings $G$ and $H$ are shown, for about three days. 


\section{Data Processing}

For the data from this site, processing was complicated by several things. First, the EMCS did not send carriage returns at the end of each line. The log file, then, often consisted of several very long lines. The second problem is that the EMCS display included a status line, which is refreshed once a minute. This line was embedded in the $\log$ file wherever the report happened to be when status line refresh occurred. Another problem is that the data are not reported in a straightforward columnar format. For COV data, a row is added to the file for each COV, consisting of one time-stamp, and one energy value. The timestamps are always in the first column, but the location of the energy value depends on which point or which date the data correspond to. Therefore, to process the data, one must be able to determine in which column the energy value is found, which is not easy to do.

In addition to processing the report file into a more readily handled data file, the data must be translated from COV format to an hourly format, for consistency with data from other buildings, and from other monitoring projects. This can be easily done using either a linear or spline fit of the data. The output of this data fitting procedure is an hourly series of cumulative values, and, just as in the Texas Tech EMCS, the data must be processed to calculate the consumption during the hour. How this is done is explained in more detail in the Appendix. Figure 13 illustrates both the COV and an hourly linear fit of the COV data for the second meter in building G. It can be seen that, for this building, little error is introduced in transforming to hourly data. The graphs presented earlier are in COV format. For building $\mathrm{F}$ which has a higher demand, (see again Figure 12a), data are recorded at a shorter interval, so that errors are even less. For building J, (Figure 12b), which has a much smaller demand, data are recorded only very infrequently, and COV data do not provide sufficient detail to convert to hourly data.

None of these are serious problems, but they make processing more difficult. We wrote AWK programs to process some of the data formats. Essentially, the programs split up long lines, extract the relevant information, and output the data into an intermediate data file. Wo defined a format for this file that would be easily read by a spreadsheet program, although almost any output format would be possible.

\section{EVALUATION}

We were able to obtain data from all three case-study sites. Each site required a slightly different method of retrieval, and none of the systems can be said to be ideal for monitoring, although each demonstrated promising capabilities. Each system had its advantages and disadvantages. The systems are compared in Table 6, according to several criteria, as discussed below. In this table, a " + " indicates a favorable evaluation of the system for that criterion, and a "-" indicates an unfavorable evaluation. One should also note that in some cases a negative evaluation is due to a limitation of the EMCS model itself, while in other cases it is due to unimplemented but available options, or other site-specific limitations. The latter is indicated with an asterisk.

\section{Trend Capacity}

All of the sites had sufficient trending capacity, at least in theory. In practice, however, both Prairie View and UTSMC are limited by communication considerations. Both Prairie View and UTSMC had several 

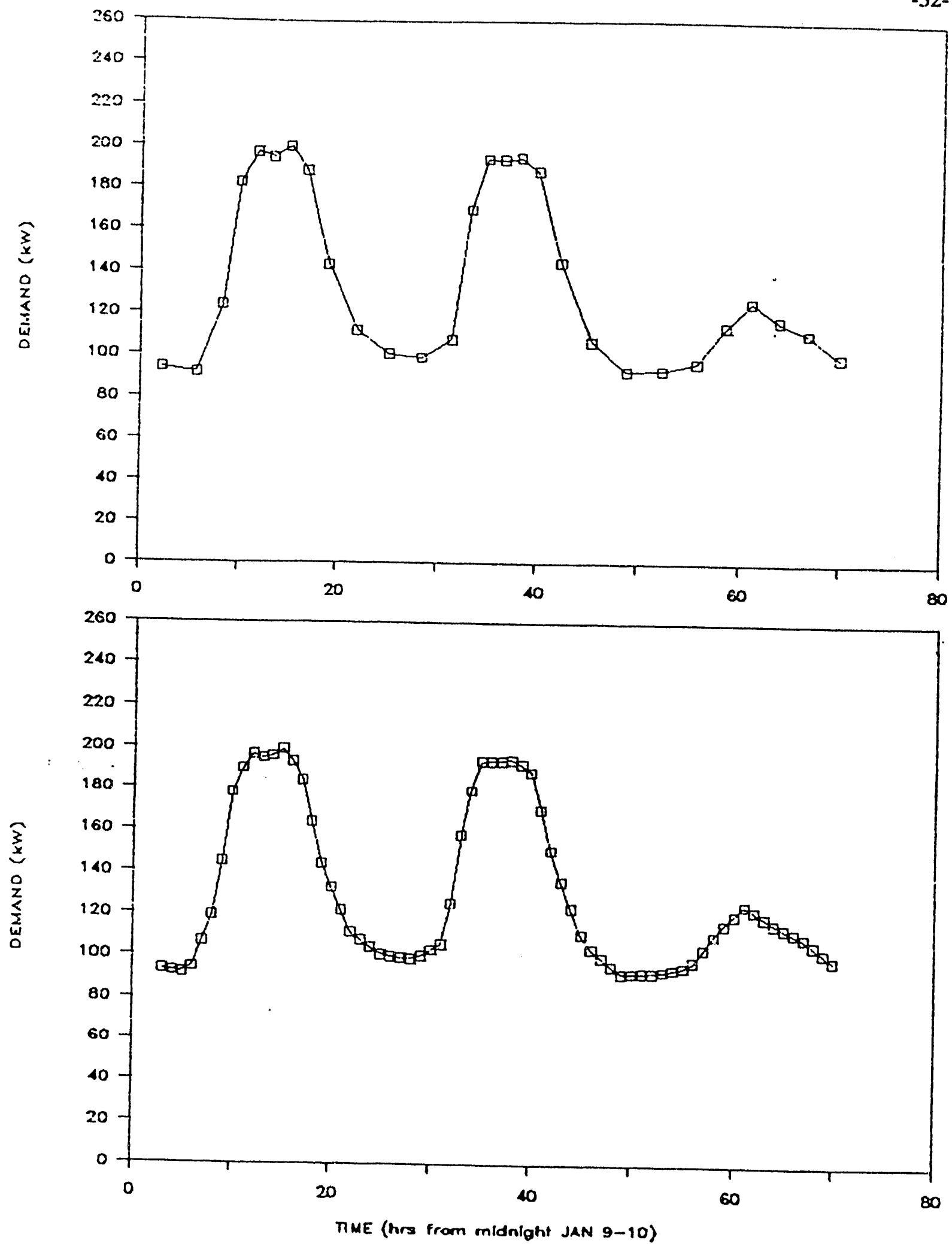

FIGURE 13-Transforming COV Data to Hourly, for UTSMC. Figure (a) shows the data in its original, COV format. Rather than collecting a datapoint each hour, it collects a datapoint when $300 \mathrm{kWh}$ have been consumed. Therefore, data are not at even intervals along the $x$-axis. In (b), a linear fit of the COV data has been performed, and hourly data have been generated from this fit. Clearly, there is little difference in the profiles. 
TABLE 6-Applicability of Case Study EMCSs for Monitoring.

\begin{tabular}{|r||c|c|c|}
\hline Site & $\begin{array}{c}\text { Prairic View } \\
\text { A\&M }\end{array}$ & $\begin{array}{c}\text { Texas Tech University } \\
\text { Health Science Center }\end{array}$ & $\begin{array}{c}\text { University of Texas } \\
\text { Southwest Medical Center }\end{array}$ \\
\hline $\begin{array}{r}\text { LMCS } \\
\text { Manufacturer }\end{array}$ & Prairie View & Lubbock & Dallas \\
\hline Model & JC/85/40(TI) & Delta 1000 & System 600 SCU \\
\hline Criterion & & Honeywell & $-*$ \\
\hline Has Sufficient Trend Capacity & $-*$ & + & + \\
\hline Appropriate Data Stored & $-*$ & $-*$ & $-*$ \\
\hline Hourly, On the Hour & + & - & - \\
\hline Access Easily Automated & + & - & - \\
\hline Short Transfer Time & + & + & + \\
\hline Error Checking Performed & - & + & - \\
\hline Data Easily Processed & + & + & + \\
\hline No Software Modifications Required & + & + & \\
\hline
\end{tabular}

* Site-specific limitation.

different ways of collecting data, due to the distributed architecture of each EMCS. These sites indicate that networking concerns may be more important considerations than raw data storage space or absolute point limits in evaluating the usefulress of an EMCS for monitoring. Availability of the trend capacity is therefore difficult to predict for a particular site by simply knowing the EMCS model.

\section{Appropriateness of Stored Data}

Beyond measuring the appropriate variables, an important consideration in EMCS monitoring is how the values are stored. All three of the systems investigated were capable of trending only instantaneous values. This is appropriate for some types of data, and not for others. For example, at UTSMC electricity consumption pulses are constantly totalized to provide a cumulative value, while at Prairie View and Texas Tech, the number of pulses received in the last minute are used to indicate "instantaneous" power. Hourly samples of cumulative energy consumption can be used to calculate hourly average energy consumption, but hourly samples of power will be quite inaccurate for reporting hourly energy use. The Prairie View EMCS had a facility for totalizing values, but it could not easily be integrated with hourly trending. Allowing for collection of data averaged over some period is an important feature for monitoring, which many EMCSs do have. 
Hourly Data, Recorded on the Hour

In order to be compatible with data from other monitoring projects and other buildings within this project, and with weather data, the data available from EMCSs should be reported at the top of each hour. The EMCS at Prairie View is capable of doing this reliably. The EMCS at Texas Tech could theoretically record data at the top of each hour, although when the system is rebooted, it will not begin collecting at the correct times, so it can be considered unreliable in this sense. The EMCS at UTSMC provides data by COV rather than by time. The data can be processed to provide hourly data values at the top of the hour. If the COV level is set correctly, little error should be introduced by doing this (see Appendix for a more complete discussion). Since COV trending has both advantages and disadvantages, EMCSs should allow the user to select which form of trending to use for each variable. More recent versions of the EMCS model used at UTSMC do allow for this facility.

\section{Easily Automated Access}

When performing case studies, it is not a problem to manually log onto an EMCS to download data. However, in a larger-scale evaluation project, a more automated method of data retrieval must be used. Most communications software packages allow the user to create a script file, which can automatically dial the phone, watch for prompts coming from the EMCS, issue the appropriate responses, and log the session. The Prairie View EMCS is very well suited to this form of automation. After dialing the phone, the script file has only to provide the correct login name, and then issue one-line commands to request the data. Interactions with the UTSMC EMCS, on the other hand, are more complex. A whole series of questions are asked, and responses must be provided to request the data. This could be automated, although it is significantly more complex than the Prairie View EMCS. The Texas Tech procedure, since it involves a human step, cannot be considered automated. From the LoanSTAR end, however, the FTP procedure could be easily automated.

\section{Short Transfer Time}

The amount of time required for transmission of the data is also an important consideration. Most EMCSs allow a dial-in connection at 1200 or 2400 baud. However, the speed of the transfer will depend to an even greater extent on the conciseness of the report format. The data from Prairie View are in a very compact form. The data from UTSMC are in a much more bulky report format, and take quite a bit longer to transmit. The data from Texas Tech are in data interchange format (DIF), which is very bulky. However, the transmission from Texas Tech takes place over a mainframe network, and at a very high transmission rate.

\section{Error Checking}

The reliability of the data collected by the EMCS is related to the reliability of the sensors and the data storage medium. These should be relatively high. However, errors can occur when transmitting data from the site to LoanSTAR. With both Prairie View and UTSMC, data are displayed on our computer screen, and simultaneously stored in a log file. No form of error checking takes place, and any noise in the phone lines can obliterate data, or change values. At Texas Tech, the data are transmitted over a computer network, using a standardized file transfer protocol, FTP. This protocol includes both error checking 
and correction, so transfer will be quite reliable.

\section{Easily Processed}

The convention for data in the LoanSTAR database is to have one file for each datalogger, including one line for each hour. Each line contains a time-stamp, and data values for several different points. EMCSs should be capable of providing data in this format, or the data should be able to be processed into this format. The Texas Tech data are reported in an acceptable format, and only minor processing had to be done, such as parsing the date into day, month, and year, and subtracting cumulative values to obtain hourly values. With the Prairie View system, the data must be processed to calculate a time-stamp, and put data in columnar form. UTSMC data requires sophisticated processing, due to several factors: only 80 columns of data are displayed, carriage returns are not transmitted, a status line can interrupt the data at any time, the data are not in conventional columnar format, and the data are in COV rather than hourly format. All of these can be dealt with, but when taken together, make data collection cumbersome.

\section{No Additional Hardware Required}

Aside from a few sensors at Texas Tech, no hardware was added at any of the sites. Monitoring would have been greatly facilitated at UTSMC and at Prairie View if a PC were purchased and added to the system. However, one of the key advantages of EMCS monitoring is that it is capable of making use of existing hardware. Most of the difficulties encountered in this project were related to easily remedied software problems, and few problems with existing EMCS hardware were encountered.

\section{No Software Modifications Required}

Software modifications were made at only one of the sites. UTSMC required no reconfiguration, and Prairie View required a minor configuration change. However, for all sites relatively minor changes to the software would have greatly facilitated monitoring. For Prairie View, it would have been much easier to set up monitoring if the software allowed the trend reports to be printed to the screen, or if it allowed the system to have more than one type of dial-in computer. Texas Tech also would benefit from being able to print the data to the screen, or to be able to transfer a disk file using a file transfer protocol, such as FTP or Kermit. UTSMC would require the least-significant modifications, including the ability to store data by hour as well as by COV (their more recent models do allow this), and a more streamlined report procedure.

\section{CONCLUSIONS}

Using in-place EMCSs for building performance monitoring has many potential advantages over conventional dedicated monitoring. The primary advantage is its cost, which could be on the order of one half to one tenth the cost of dedicated monitoring, or even less. Additional advantages are found in the additional data available to the researcher, the computation capabilities of the EMCS itself, and the potential for immediate feedback to the building operators. We were able to use the EMCSs in three different sites to collect performance data. However, at each site, issues arose making development of the data collection procedures more complex. 
Relatively minor modifications to the available EMCS software could greatly improve this method of collecting data. In particular, EMCS software should be modified to allow data to be averaged over an hourly interval and reliably reported at the end of each hour, create concise and consistent formats for requesting and reporting the data, and create some simple means of displaying or transmitting the data.

The simplest way for us to gain access to the data in these existing cases was to have the data displayed on the screen and captured into a log file. However, this is not the most appropriate method, due to the inability to perform error detection and correction, and the time it takes to transmit and process the report. Ideally, controls manufacturers should incorporate into their basic software a procedure for transmitting data files to a remote, dial-in terminal, using a standard file transfer protocol.

One means of encouraging controls manufacturers to make these modifications would be if a major monitoring program, such as LoanSTAR, were to create specifications for EMCS monitoring capabilities. Whenever an EMCS is specified as a conservation measure, it should be used for monitoring, and, to do so, it must meet some minimal monitoring specifications. Since this would favor selection of EMCSs that meet these specifications, it would provide EMCS manufacturers with an incentive to make the required software madifications.

With co-funding from the Department of Energy (DOE), we are working on identifying and outlining EMCS-based monitoring specifications. Future collaboration between LoanSTAR and DOE will help meet the LoanSTAR objectives of developing inexpensive, powerful, and reliable methods of performance monitoring.

\section{ACKNOWLEDGEMENTS}

This work was sponsored by the Texas Governor's Energy Management Center, as part of the LoanSTAR Monitoring and Analysis Project, through Texas A\&M University (Award No. BG90319); and by the Assistant Secretary for Conservation and Renewable Energy, Office of Building Technology of the U.S. Department of Energy, under Contract No. DE-AC03-76SF00098.

The authors gratefully acknowledge the EMCS operators and manufacturers representatives who provided valuable assistance in this project; specifically Scott Moomaw and Keith Taconi (Houston), and Norman Ferguson and Ed Bruner (Prairie View) with Johnson Controls, Inc.; Vernon Linton with Texas Tech University Health Science Center; and Tony Welton with the University of Texas Southwest Medical Center. We also acknowledge the assistance of other staff of the LoanSTAR program, including John Bryant, Robert Sparks, and Robert Lopez.

\section{REFERENCES}

Akbari, H., K. Heinemeier, and M. Goralka. 1989. Energy Management and Control Systems for Utility Load Monitoring and Real-Time Pricing. LBL-26689. 
Flora, D., P. LeConiac, and H. Akbari. 1986. "Energy Management Systems-An Emerging Method to Obtain Building Energy Performance Data." Proceedings of Energy Management Conference. Los Angeles, CA.

Heinemeier, K., and H. Akbari. 1987. "Capabilities of In-Place Energy Management Systems for Remote MOnitoring of Building Energy Performance-Case Studies." ASHRAE Transactions. Vol. 93, Part 2.

LeConiac, P., D. Flora, and H. Akbari. 1986. "Energy Management Systems as a Source of Building Energy Performance Data." ACEEE 1986 Summer Study on Energy Efficiency in Buildings, Vol. 9.

\section{APPENDIX: COV MONITORING}

Change of value monitoring (as implemented at the UTSMC site) is an innovative way of collecting data, since it collects the most data when things are likely to be most interesting. When COV's are used with cumulative energy data, they collect the most data during peak hours, which may be of the greatest interest for utilities. If COV's were used with instantaneous power, they would record the most data during shoulder hours, when things are changing. This may be of the greatest interest to building researchers. However, it is a data format that is not familiar to most building energy researchers, and is not compatible with most other building and weather data.

Can COV data be transformed to hourly data without introducing significant error? To analyze the impact of using COV data, we wrote several programs to easily generate COV and hourly data files. The first program reads in a one-minute data file and produce cumulative data in both hourly and COV formats. The hourly file contains hourly samples of cumulative energy consumption, as one would expect to find from many EMCSs. The COV file contains a sample of cumulative energy consumption every time the user-defined COV value has been reached; for example, the time and cumulative energy consumption are recorded whenever $300 \mathrm{kWh}$ have been consumed. Another program then reads these files and calculates hourly average power values. The real hourly file is simply the difference between two consecutive cumulative energy readings. The COV-hourly file uses a linear fit to calculate hourly energy consumption. Ideally, these would be identical for both hourly and COV data.

We used synthetic data to test and evaluate the applicability of COV data. The one-minute data file was a sinusoid, with a period of 24 hours, consisting of 1440 values per day. Hourly data were generated from the one-minute data, and are shown in Figure 14 as squares. COV data were then generated, for several COV levels. Hourly data were then generated from linear fits of the COV data. The graph indicates a close comparison between the real hourly data and the COV-hourly data so long as the COV level is less than about twice the hourly energy consumption. This corresponds to one sample every two hours (for example, $250 \mathrm{~kW}$ divided by $500 \mathrm{kWh} / \mathrm{sample}$ is 1 sample in two hours). For higher and higher COV values, there is a larger and larger discrepancy between the two.

Figure 15 shows this discrepancy as a function of the current sampling rate, for three different COV levels, and over 24 hours. For example, when the COV is set to be equal to the peak hourly consumption levil (diamonds, in Figure 15), the effective sampling rate reaches a maximum of one sample per hour 


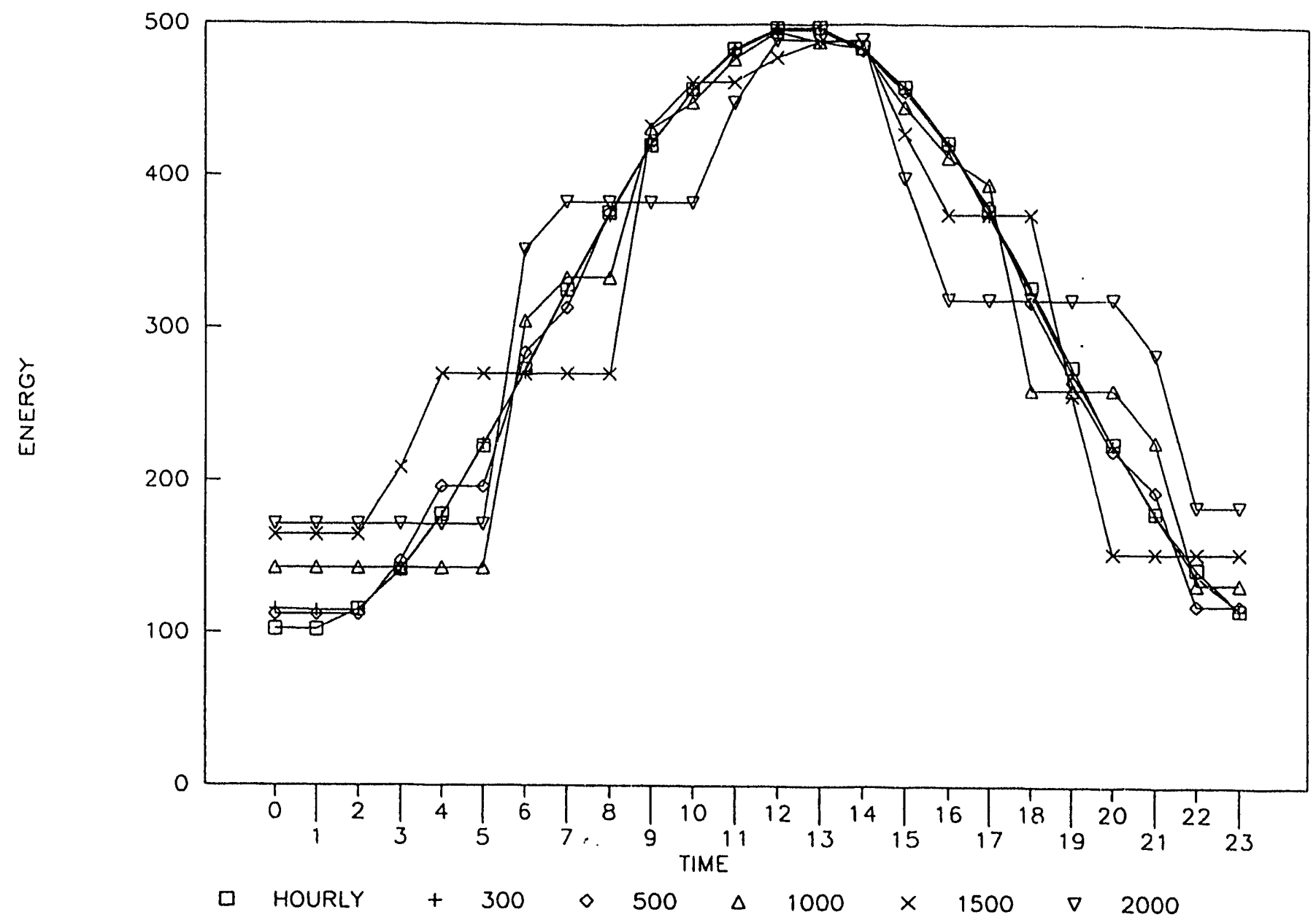

FIGURE 14-Analysis of the Effect of the COV Level. For sinusoidal synthetic data, an hourly profile was created (squares). COV data were also generated from the sinusoidal data, using several different COV levels. A linear fit was performed on the COV data, and hourly data were generated, shown here for the different COV levels. When the COV level was low relative to the peak demand (COV $=300$, crosses) little deviation is found from the original hourly data. For higher COV levels, a greater deviation is found, and the profile is distorted. 
during piak periods. However, during the nighttime, data are coming in much less frequently, and the discrepancy between this COV data and hourly averaging can be fairly high. When the COV is set to be equal to the minimum consumption level (squares, in Figure 15), at least one sample is recorded each hour, so there is never a discrepancy of more than two percent. However, during peak periods the sampling rate increases, up to almost five samples per hour. This may or may not cause communications problems. It is interesting also to look at an intermediate COV level (crosses, in Figure 15). In this case the sampling rate is never greater than two samples per hour, and the error is almost always less than two percent. The few hours with larger errors occur in the middle of the night, which may be acceptable for some analysis.

Clearly, the COV value is an extremely important figure-it determines whether the communications lines will be overloaded with data, or whether only one or two data points will be collected each day, or something appropriately in between these extremes. The setting of this value is probably the most important determinant of whether or not COV data collection is appropriate for energy monitoring. Yet, how is the value determined? We are not sure yet how it is typically done, but it seerns clear that during the EMCS commissioning period, this value should be monitored and evaluated, and an analysis similar to ours should be done. 


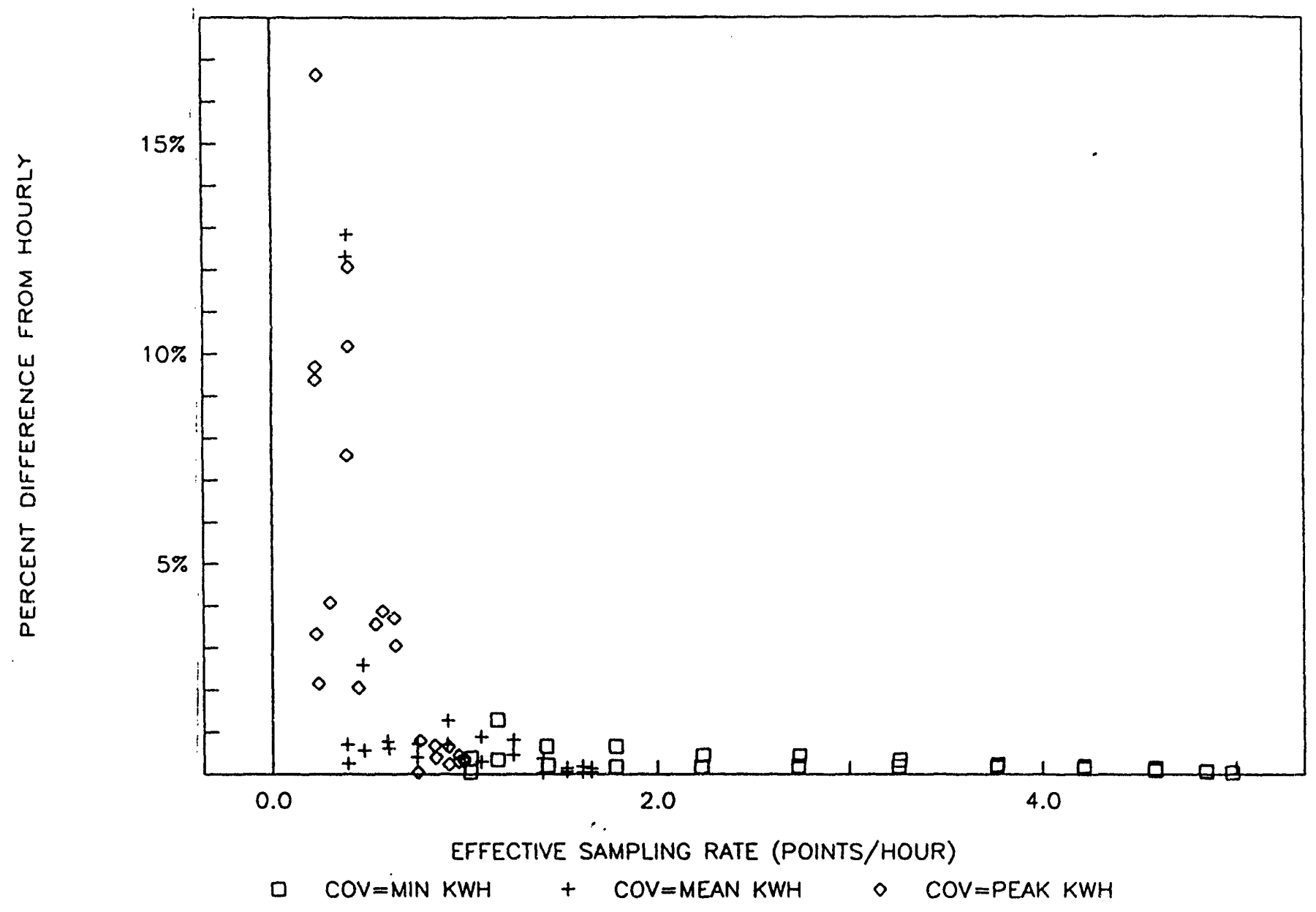

FIGURE 15-Errors Introduced by Using COV Data. For 24 hours, the percent (absolute value) difference between hourly data and hourly data generated from COV data, are shown versus the sampling rate, for different COV levels. With a low COV level, (for example, equal to the minimum demand rate-squares), very little error is introduced, although the relatively high sample rate may cause problems with communications. With a high COV level, (equal to the maximum demand rate-diamonds), the sampling rate is reduced, but errors are introduced. An intermediate COV level, (equal to the mean demand rate-crosses), results in a good compromise: low sampling rate, and low enrors. The few times higher errors do oocur for this COV level are during the middle of the night, and may be acceptable. 

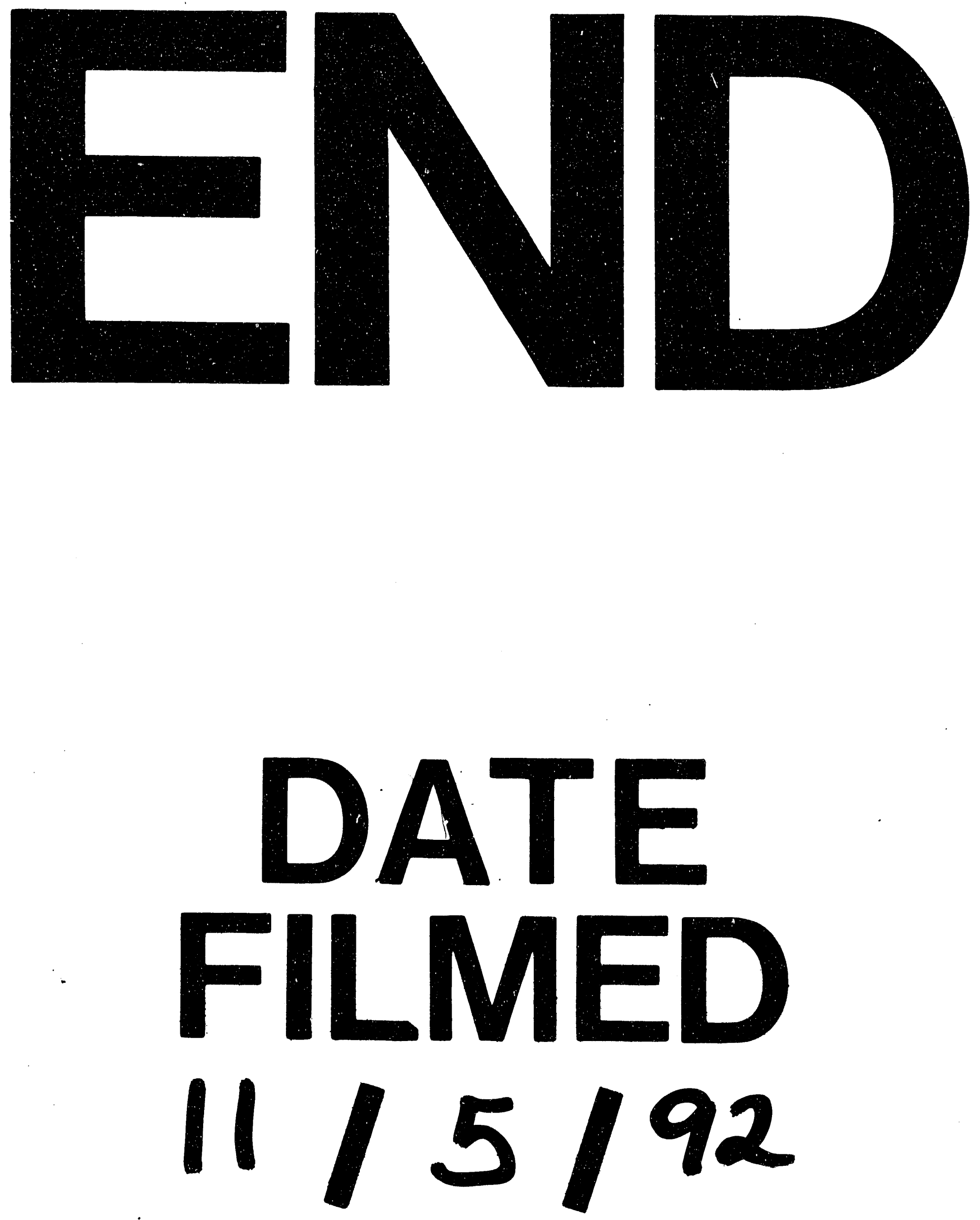

1 
